\title{
ABSTRACT MECHANICAL CONNECTION AND ABELIAN RECONSTRUCTION FOR ALMOST KÄHLER MANIFOLDS
}

\author{
SERGEY PEKARSKY AND JERROLD E. MARSDEN
}

Received 12 September 2000 and in revised form 20 February 2001

When the phase space $P$ of a Hamiltonian G-system $(P, \omega, G, J, H)$ has an almost Kähler structure, a preferred connection, called abstract mechanical connection, can be defined by declaring horizontal spaces at each point to be metric orthogonal to the tangent to the group orbit. Explicit formulas for the corresponding connection one-form $\mathcal{A}$ are derived in terms of the momentum map, symplectic and complex structures. Such connection can play the role of the reconstruction connection (due to the work of A. Blaom), thus significantly simplifying computations of the corresponding dynamic and geometric phases for an Abelian group G. These ideas are illustrated using the example of the resonant three-wave interaction. Explicit formulas for the connection one-form and the phases are given together with some new results on the symmetry reduction of the Poisson structure.

\section{Introduction}

\subsection{Definitions and preliminaries}

Consider a finite-dimensional symplectic manifold $(P, \omega)$. Let a Lie group $G$ act on it canonically, that is, by preserving the symplectic form $\omega$, and assume that this action admits an (equivariant) momentum map $\mathrm{J}: \mathrm{P} \rightarrow \mathrm{U} \subset$ $\mathfrak{g}^{*}, \mathrm{U} \equiv \mathrm{J}(\mathrm{P})$. Let a dynamical system be defined on $\mathrm{P}$ by some Hamiltonian $\mathrm{H}$. We call $(P, \omega, G, J, H)$ a Hamiltonian G-system. Assume also that $G$ acts on $P$ freely and properly so that the Poisson reduction can be performed (in fact, these conditions can be slightly relaxed, cf. [9]). For background on momentum maps, Poisson reduction, etc., the reader is referred to Marsden and Ratiu [9]. 
2 Abstract mechanical connection and Abelian reconstruction ...

Recall that an almost Kähler manifold $(M, \omega, \mathcal{J}, \mathfrak{s})$ can be defined as a manifold $M$ with an almost complex structure $\mathcal{J}$ and a $\mathcal{J}$-invariant (i.e., Hermitian) metric $\mathfrak{s}$, whose fundamental 2-form $\omega$, defined by

$$
\omega(x)(\boldsymbol{v}, \boldsymbol{w})=\mathfrak{s}(x)(\mathcal{J}(x) \boldsymbol{v}, \boldsymbol{w}), \quad \forall v, w \in \mathrm{T}_{\chi} M
$$

is closed and hence a symplectic form on $M$. If in addition the Nijenhuis torsion of $\mathcal{J}$ vanishes, then $\mathcal{J}$ is complex and $M$ becomes a Kähler manifold [6]. The automorphisms of an almost Kähler structure are diffeomorphisms of $M$ which at the same time are symplectomorphisms, almost complex maps and isometries with respect to $\omega, \mathcal{J}$, and $\mathfrak{s}$, respectively. It follows from the definition that any two of these conditions imply the third one. For the background and more information, see, for example, Kobayashi and Nomizu [6].

\subsection{Reconstruction of the dynamics}

The space of group orbits $P / G$, which is obtained by taking the quotient map $\pi: \mathrm{P} \rightarrow \mathrm{P} / \mathrm{G}$ and is a smooth manifold under appropriate assumptions, inherits a Poisson structure from that of $\mathrm{P}$. The Hamiltonian $\mathrm{H}$ drops to a reduced Hamiltonian $h$ on $P / G$, and the corresponding Hamiltonian vector fields $X_{H}$ and $X_{h}$, as well as their solutions $x_{t}$ and $y_{t}$, respectively, are related by the projection $\pi: \mathrm{P} \rightarrow \mathrm{P} / \mathrm{G}$.

Assume that $y_{t}$ is periodic with period $T$, then for any initial condition $x_{0} \in \pi^{-1}\left(y_{0}\right)$, the associated reconstruction phase is the unique $g \in G$ such that $x_{T}=g \cdot x_{0}$. The methods presently used to compute reconstruction phases are generally based on those established in [8]. The procedure can be sketched as follows.

If $\mathrm{J}: \mathrm{P} \rightarrow \mathfrak{g}^{*}$ is a momentum map, which we will suppose is $\mathrm{Ad}^{*}$ equivariant, then $\mathrm{J}\left(\mathrm{x}_{\mathrm{t}}\right)=\mu_{0} \equiv \mathrm{J}\left(\mathrm{x}_{0}\right)$. Under appropriate connectedness hypotheses, the Marsden-Weinstein reduced space $J^{-1}\left(\mu_{0}\right) / G_{\mu_{0}}\left(G_{\mu_{0}}\right.$ denoting the isotropy of the coadjoint action at $\left.\mu_{0} \in \mathfrak{g}^{*}\right)$ can be identified with a symplectic leaf $P_{\mu_{0}} \subset P / G$ containing the reduced solution curve $y_{t}$, and the projection $\mathrm{J}^{-1}\left(\mu_{0}\right) \rightarrow \mathrm{P}_{\mu_{0}}$ is a principal $G_{\mu_{0}}$-bundle.

The first step in calculating the reconstruction phase $\mathrm{g}$ is to equip the bundle $\mathrm{J}^{-1}\left(\mu_{0}\right) \rightarrow \mathrm{P}_{\mu_{0}}$ with a principal connection $\alpha_{\mu_{0}}$, whose holonomy along the reduced curve $y_{t}$ is called the associated geometric phase and denoted $g_{\text {geom }}$. The phase $g$ is then the product $g_{\text {dyn }} g_{\text {geom }}$, where $g_{\text {dyn }}$, called the dynamic phase, is obtained by integrating a linear, nonautonomous, and ordinary differential equation, called the reconstruction equation. The coefficients in this equation are defined in terms of $\alpha_{\mu_{0}}$, the unreduced Hamiltonian vector field $X_{H}$, and an $\alpha_{\mu_{0}}$-horizontal lift of $y_{t}$ to $J^{-1}\left(\mu_{0}\right)$. Calculating the geometric phase usually requires one to compute the curvature of $\alpha_{\mu_{0}}$. 
While any connection $\alpha_{\mu_{0}}$ can be used to compute $g$ as described in the previous paragraph, a poor choice will lead to unwieldy computations (see, for example, [11]). For the so-called simple mechanical G-systems a natural choice exists (see Section 1.3); for other systems the choice is often made on a case-by-case basis.

\subsection{Overview of the results}

As we already mentioned, the methods presently used to compute reconstruction phases are based on those established in [8]. Though the general ideas in [8] apply for arbitrary Hamiltonian G-systems, most of the advances in the computation techniques have been done for mechanical systems on cotangent bundles $T^{*} \mathrm{Q}$ of some Riemannian manifolds $\mathrm{Q}$ with the metric, which determines the kinetic energy, playing the crucial role for the definition of the mechanical connection. Unfortunately, these settings exclude such interesting and important systems as $\mathrm{N}$ point vortices on a plane or on a sphere, $\mathrm{N}$-wave interaction, etc., where the configuration space is not a cotangent bundle.

Luckily, some of these systems have a natural almost Kähler structure which we exploit in the construction of the abstract mechanical connection. It is defined by specifying the horizontal space to be metric orthogonal to the group orbits (see Section 4 for the details). The corresponding connection one-form is then obtained in terms of the momentum map, symplectic and complex structures. These expressions enable us to further simplify the computation of the reconstruction phases as described in [3] for the case of Abelian groups. The requirement of the group being Abelian is essential for the geometric phase part (see Section 4.2), but it is not used in the construction of the map L involving the abstract inertia tensor and in the expression for the dynamic phase. The Abelian property of the group makes the relation between the principal connections on Poisson and symplectic bundles trivial (see Section 3.1). It also significantly simplifies the picture of dual pairs, which underlies our constructions, and enables us to construct a very "useful" bundle $j: P / G \rightarrow \mathfrak{g}^{*}$. This is briefly described in Section 3.2.

In the work in progress a generalization to non-Abelian group action is being considered, as well as further simplification and links which arise in the case of the phase space being a cotangent bundle with the almost Kähler structure coming from a Riemannian metric on the configuration space. The relation between the abstract mechanical connection and the well-known mechanical connection is considered in [3], where the reconstruction phases for the cotangent bundles were analyzed, though not from the point of view of almost Kähler manifolds and corresponding abstract mechanical connections. 
4 Abstract mechanical connection and Abelian reconstruction ...

\section{Reconstruction connection and the associated phases}

In this section, we briefly overview the results on reconstruction phases obtained in [3]. We refer the reader to the original paper for detailed and comprehensive treatment of the subject. Here we are mainly interested in adopting these results to the case of almost Kähler systems, and thus we avoid giving much details to keep the presentation clear and avoid repetition.

In [3], a general formula is derived which expresses a reconstruction phase in terms of the associated reduced solution, viewed as a curve in the Poissonreduced phase space $P / G$, and certain derivatives transverse to the symplectic leaf in $P / G$ containing the curve. Specifically, the dynamic part of the phase depends on transverse derivative in the Poisson-reduced Hamiltonian, while the geometric part is determined by transverse derivatives in the leaf symplectic structures.

\subsection{Highlights and basic assumptions}

It is shown in [3] that the principal connection on the bundle $\mathrm{J}^{-1}\left(\mu_{0}\right) \rightarrow$ $\mathrm{P}_{\mu_{0}}$, which plays a crucial role in the computation of the phases, is most naturally viewed as the restriction to $\mathrm{J}^{-1}\left(\mu_{0}\right)$ of a certain kind of distribution $A$ on $P$, which is called a reconstruction connection. To define the transverse derivatives, one then specifies a connection $\mathrm{D}$ on the symplectic stratification of $\mathrm{P} / \mathrm{G}$ (a distribution on $\mathrm{P} / \mathrm{G}$ furnishing a complement for the characteristic distribution). This connection $\mathrm{D}$ can be obtained by "Poisson-reducing" the connection $A$.

Explicitly, assuming that as a cycle, the reduced curve $y_{t}$ (see Section 1.2) is a boundary $\partial \Sigma\left(\Sigma \subset \mathrm{P}_{\mu_{0}}\right.$ compact and oriented), the corresponding reconstruction phase is $\mathrm{g}=\mathrm{g}_{\text {dyn }} \mathrm{g}_{\text {geom }}$, where

$$
g_{d y n}=\exp \int_{0}^{T} D_{\mu_{0}} h\left(y_{t}\right) d t, \quad g_{g e o m}=\exp \int_{\Sigma} D_{\mu_{0}} \omega_{D}
$$

In these formulas $\mathrm{D}_{\mu_{0}}$ denotes a certain "exterior covariant derivative" depending on $D$ and $\mu_{0}$ that maps $\mathbb{R}$-valued $p$-forms on $P / G$ to $\mathfrak{g}_{\mu_{0}}$-valued $p$-forms on $P_{\mu_{0}}(p=0,1,2, \ldots)$. For example, $D_{\mu_{0}} h\left(y_{t}\right)$ is an element of $\mathfrak{g}_{\mu_{0}}$ that happens to measure the derivative of $h$ in directions lying in $\mathrm{D}\left(\mathrm{y}_{t}\right)$ (i.e., in certain directions transverse to the symplectic leaves). $\omega_{D}$ denotes the two-form on $\mathrm{P} / \mathrm{G}$ whose restriction to a given leaf gives that leaf's symplectic structure, and whose contraction with vectors in $\mathrm{D}$ vanishes.

Equation (2.1) holds assuming that $G_{\mu_{0}}$ is Abelian, that $P_{\mu_{0}}$ is a nondegenerate symplectic leaf, and that $\mathrm{D}$ is a smooth distribution in some neighborhood of $P / G$. These conditions are in addition to the following assumptions which are understood to be in place throughout the paper:

- all manifolds are smooth, that is, $\mathrm{C}^{\infty}$ 
- the group $\mathrm{G}$ acts freely and properly, so that the natural projection $\pi: \mathrm{P} \rightarrow \mathrm{P} / \mathrm{G}$ is a submersion

- the group action is Hamiltonian, that is, it admits a momentum map $\mathrm{J}: \mathrm{P} \rightarrow \mathfrak{g}^{*}$ which is $\mathrm{Ad}^{*}$-equivariant, that is, $\mathrm{J}(\mathrm{g} \cdot \mathrm{x})=\mathrm{Ad}_{\mathrm{g}}^{*} \mathrm{~J}(\mathrm{x})$.

Expressions (2.1) make sense for any connection $\mathrm{D}$ on the symplectic stratification of $\mathrm{P} / \mathrm{G}$; whence, the total reconstruction phase $\mathrm{g}=\mathrm{g}_{\text {dyn }} \mathrm{g}_{\text {geom }}$ (which is independent of the choice of $\alpha_{\mu_{0}}$, and hence $A$ ) can be computed using any connection $\mathrm{D}$ on the symplectic stratification of $\mathrm{P} / \mathrm{G}$.

The following two subsections give a short review of the results in [3] that are relevant for our applications.

\subsection{Main constructions}

Definition 2.1. Call a distribution $A$ on $\mathrm{P}$ a reconstruction connection if

(a) $A$ is G-invariant,

(b) $\operatorname{Ker} T_{x} J=T_{x}\left(G_{\mu} \cdot x\right) \oplus A(x)(x \in P, m \equiv J(x))$.

Here $G_{\mu}$ denotes the point stabilizer of the coadjoint action at $\mu \in \mathfrak{g}^{*}, T_{x} J$ is the tangent map, and $\oplus$ denotes the direct sum.

\subsubsection{Connections on the symplectic stratification of $\mathrm{P} / \mathrm{G}$}

Let $E$ denote the characteristic distribution on $P / G$ (i.e., the distribution tangent to the symplectic leaves). We call a distribution $\mathrm{D}$ on $\mathrm{P} / \mathrm{G}$ a connection on the symplectic stratification of $\mathrm{P} / \mathrm{G}$ if it furnishes a complement for E:

$$
\mathrm{T}(\mathrm{P} / \mathrm{G})=\mathrm{E} \oplus \mathrm{D} .
$$

Now let $A$ be a G-invariant distribution on $P$. Since $G$ acts by symplectic diffeomorphisms, the distribution $A^{\omega}$, the symplectic orthogonal distribution to $A$, is also $\mathrm{G}$-invariant. It consequently drops to a distribution $\widehat{A} \equiv \pi_{*}\left(A^{\omega}\right)$ on $\mathrm{P} / \mathrm{G}$; here $\pi_{*}$ denotes push-forward. Conversely, if $\mathrm{D}$ is an arbitrary distribution on $\mathrm{P} / \mathrm{G}$, then $\widehat{\mathrm{D}} \equiv\left(\pi^{*} \mathrm{D}\right)^{\omega}$ is a G-invariant distribution on $\mathrm{P}$; here $\pi^{*}$ denotes pull-back. Evidently, one has

$$
\widehat{\mathrm{D}}=\mathrm{D} \text {. }
$$

We quote the following theorem form [3] without proof.

Theorem 2.2 (Blaom 1999). If $A$ is a general reconstruction connection, then $\widehat{A}$ is a connection on the symplectic stratification of $\mathrm{P} / \mathrm{G}$. Moreover, the map $A \mapsto \widehat{A}$ is a bijection from the set of reconstruction connections to the set of connections on the symplectic stratification of $\mathrm{P} / \mathrm{G}$. This bijection has an inverse $\mathrm{D} \mapsto \widehat{\mathrm{D}}$. 
6 Abstract mechanical connection and Abelian reconstruction ...

If $A$ is a reconstruction connection, one thinks of $\widehat{A}$ as its Poisson-reduced counterpart. A reconstruction connection $A$ can be reconstructed from its reduced counterpart $D \equiv \widehat{A}$ according to $A=\widehat{D}$.

Two other lemmas from [3] are relevant to our presentation and will be used in Section 3.

Lemma 2.3. Let $\pi: \mathrm{P} \rightarrow \mathrm{Q}$ be a Poisson submersion and let $\mathrm{E}$ denote the characteristic distribution on $\mathrm{Q}$. If $\mathrm{P}$ is symplectic, and $\omega$ denotes the symplectic form on $\mathrm{P}$, then

$$
\pi^{*} \mathrm{E}=\operatorname{Ker} \mathrm{T} \pi+(\operatorname{Ker} \mathrm{T} \pi)^{\omega} .
$$

Lemma 2.4. Let $x \in \mathrm{P}$ be arbitrary and define $\mu \equiv \mathrm{J}(\mathrm{x})$. Then

$$
\mathrm{T}_{\chi}\left(\mathrm{G}_{\mu} \cdot x\right)=\left(\left(\pi^{*} \mathrm{E}\right)(x)\right)^{\omega}
$$

\subsubsection{Transverse derivatives in $\mathrm{P} / \mathrm{G}$}

Under appropriate connectedness hypotheses each reduced space $J^{-1}(\mu) / G_{\mu}$ may be identified with a symplectic leaf $P_{\mu} \subset P / G$. A connection $D$ on the symplectic stratification of $P / G$ allows one to define derivatives of functions on $P / G$ transverse to $P_{\mu}$. At a point in $P_{\mu}$ such a derivative can be identified in a natural way with an element of the isotropy algebra $\mathfrak{g}_{\mu}$, provided that the isotropy group $G_{\mu}$ is Abelian. More generally, for such $\mu$ the connection $\mathrm{D}$ defines an "exterior covariant derivatives" mapping $\mathbb{R}$-valued $p$-forms on $\mathrm{P} / \mathrm{G}$ to $\mathfrak{g}_{\mu}$-valued $p$-forms on the leaf $\mathrm{P}_{\mu}$.

Let $\mathrm{D}$ be a fixed connection on the symplectic stratification of $P / G$. Fix $\mu \in \mathrm{U} \equiv \mathrm{J}(\mathrm{P})$ and assume $\mathrm{G}_{\mu}$ is Abelian. Then we have the following proposition.

Proposition 2.5. For each $\mathrm{y} \in \mathrm{P}_{\mu}$ there is a natural isomorphism $\mathrm{D}(\mathrm{y}) \leftrightarrow$ $\mathfrak{g}_{\mu}^{*}$ well defined by

$$
v \longmapsto p_{\mu}(\operatorname{forg}(\mathrm{TJ} \cdot w))
$$

where $w$ denotes any element of $\mathrm{T}_{\mathrm{x}} \mathrm{P}$ with $\mathrm{T} \pi \cdot w=v$, and $x \in \mathrm{J}^{-1}(\mu) \cap$ $\pi^{-1}(y)=\pi_{\mu}^{-1}(y)$ is arbitrary. The inverse of this map (which depends on $\mathrm{D}, \mu$, and $\mathrm{y})$ is denoted by $\mathrm{L}(\mathrm{D}, \mu, \mathrm{y}): \mathfrak{g}_{\mu}^{*} \rightarrow \mathrm{D}(\mathrm{y})$.

The map forg: TU $\rightarrow \mathfrak{g}^{*}$ denotes the map that "forgets base point" and $p_{\mu}: \mathfrak{g}^{*} \rightarrow \mathfrak{g}_{\mu}^{*}$ denotes the natural projection.

Definition 2.6. Suppose that $f$ is a function on $P / G$ defined in some neighborhood of $y$. Then the $(D, \mu)$-exterior covariant derivative of $f$ at $y$, 
denoted $D_{\mu} f(y) \in \mathfrak{g}_{\mu}$, is defined through

$$
\left\langle v, D_{\mu} f(y)\right\rangle=\langle d f, L(D, \mu, y)(v)\rangle \quad \forall v \in \mathfrak{g}_{\mu}^{*} .
$$

Definition 2.7. Let $\sigma$ be a differential $p$-form on $P / G$ defined in a neighborhood of $P_{\mu}$, and assume that $G_{\mu}$ is Abelian. Then the $(D, \mu)$-exterior covariant derivative $D_{\mu} \sigma$ of $\sigma$ is the $\mathfrak{g}_{\mu}$-valued $p$-form on $P_{\mu}$ defined through

$$
\left\langle v, D_{\mu} \sigma\left(v_{1}, \ldots, y_{p}\right)\right\rangle=d \sigma\left(L(D, \mu, y)(v), v_{1}, \ldots, v_{p}\right)
$$

where $v \in \mathfrak{g}_{\mu}^{*}, v_{1}, \ldots, v_{p} \in T_{y} P_{\mu}$ and $y \in P_{\mu}$.

\subsubsection{Smoothness conditions}

Let $A$ be a reconstruction connection and let $D$ be a connection on the symplectic stratification of $P / G$. Then we say that, $A$ is $\mu$-smooth $(\mu \in U$ ) if the set

$$
\left\{A(x) \mid x \in J^{-1}(\mu)\right\}
$$

is a smooth sub-bundle of the tangent bundle $\mathrm{T}\left(\mathrm{J}^{-1}(\mu)\right)$. We call $\mathrm{D} \mu$-smooth if the set

$$
\left\{\mathrm{D}(\mathrm{y}) \mid \mathrm{y} \in \mathrm{P}_{\mu}\right\}
$$

is a smooth sub-bundle of $T_{P_{\mu}}(P / G) \equiv\left\{T_{y}(P / G) \mid y \in P_{\mu}\right\}$.

Then, the following smoothness results hold [3]:

- $\mathrm{D}$ is $\mu$-smooth if and only if $A$ is $\mu$-smooth.

- If $D$ is $\mu$-smooth, then $L(D, \mu, y)$ in (2.6) depends smoothly on $y \in P_{\mu}$.

- If $D$ is $\mu$-smooth, then $D_{\mu} f: P_{\mu} \rightarrow \mathfrak{g}_{\mu}$ is smooth.

- Similarly, for a $p$-form $\sigma, \mu$-smoothness of $D$ ensures smoothness of $\mathrm{D}_{\mu} \sigma$.

\subsection{Reconstruction phases}

Let $\mathrm{H}$ be a $\mathrm{G}$-invariant Hamiltonian on $\mathrm{P}$, and let $\mathrm{h}: \mathrm{P} / \mathrm{G} \rightarrow \mathbb{R}$ be its Poissonreduced counterpart. With the assumptions stated in Section 2.1 satisfied, consider an integral curve $x_{t} \in P$ of $X_{H}$. The curve remains in the submanifold $J^{-1}\left(\mu_{0}\right)\left(\mu_{0} \equiv J\left(x_{0}\right)\right)$ for all time $t$ for which it is defined. The Marsden-Weinstein reduction bundle

$$
\pi_{\mu_{0}}: J^{-1}\left(\mu_{0}\right) \longrightarrow P_{\mu_{0}}
$$

is a principal $G_{\mu_{0}}$-bundle. Let

$$
\alpha_{\mu_{0}}: \mathrm{T}\left(\mathrm{J}^{-1}\left(\mu_{0}\right)\right) \longrightarrow \mathfrak{g}_{\mu_{0}}
$$


8 Abstract mechanical connection and Abelian reconstruction ...

denote the connection one-form on this bundle whose associated horizontal space at each $x \in J^{-1}\left(\mu_{0}\right)$ is hor $\mu_{\mu_{0}} \equiv A(x)$. To ensure that $\alpha_{\mu_{0}}$ and hor $\mu_{0}$ are smooth, we require that $A$ be $\mu_{0}$-smooth.

Let $y_{t} \in P_{\mu_{0}}$ denote the integral curve of the reduced Hamiltonian vector field $X_{h}$ on $P / G$ that has $y_{0}=\pi\left(x_{0}\right) \in P_{\mu_{0}}$ as its initial point. Then as $X_{H}$ and $X_{h}$ are $\pi$-related, we have $y_{t}=\pi\left(x_{t}\right)$ for all $t$.

Let $d_{t} \in J^{-1}\left(\mu_{0}\right)$ denote the hor $\mu_{0}$-horizontal lift of $y_{t}$ having $x_{0}$ as its initial point $d_{0}$. Supposing that $y_{t}$ is periodic with period $T$, we have

$$
\mathrm{d}_{\mathrm{T}}=\mathrm{g}_{\text {geom }} \cdot \mathrm{x}_{0}, \quad \mathrm{x}_{\mathrm{T}}=\mathrm{g}_{\mathrm{dyn}} \cdot \mathrm{d}_{\mathrm{T}},
$$

for some uniquely defined $g_{\text {geom }}, g_{d y n} \in G_{\mu_{0}}$ called geometric and dynamic phases associated with the reduced solution $Y_{t}$. The product $g_{\text {total }}=$ $g_{\text {geom }} g_{\text {dyn }}$ is called the total phase. It does not depend on $A=\widehat{D}$, but depends only on $y_{0}$, the flow of $X_{H}$, and the period $T$.

\subsubsection{Dynamic phases}

It is well known (see [8]) that the dynamic phase is given by the solution of the following initial value problem, known as the reconstruction equation:

$$
\dot{g}_{t}=g_{t} \xi_{t}, \quad \text { where } \xi_{t} \equiv \alpha_{\mu_{0}}\left(X_{H}\left(d_{t}\right)\right), \quad g_{0}=I d .
$$

Here $g_{t} \xi_{t}$ denotes the tangent action of $g_{t}$.

Corollary 3.6 of [3] states that, assuming $G_{\mu_{0}}$ is Abelian, the dynamic phase is given by

$$
g_{d y n}=\exp \int_{0}^{T} D_{\mu_{0}} h\left(y_{t}\right) d t .
$$

\subsubsection{Geometric phases}

Recall that the geometric phase $g_{\text {geom }}$ associated with a solution $x_{t}$ is the holonomy of a principal connection $\alpha_{\mu_{0}}$ on $\mathrm{J}^{-1}\left(\mu_{0}\right) \rightarrow \mathrm{P}_{\mu_{0}}$ along the corresponding reduced solution curve $y_{t}=\pi\left(x_{t}\right) \in P_{\mu_{0}}$. Assuming $G_{\mu_{0}}$ is Abelian, the holonomy of appropriate curves is determined by the curvature of $\alpha_{\mu_{0}}$. It is well known (see [8]) that if the cycle $y_{t}$ is in fact a boundary $\partial \Sigma\left(\Sigma \subset P_{\mu_{0}}\right.$ compact and oriented), then

$$
g_{\text {geom }}=\exp \left(-\int_{\Sigma} \Omega_{\mu_{0}}\right),
$$

where $\Omega_{\mu_{0}}$ is the curvature of $\alpha_{\mu_{0}}$, viewed as a $\mathfrak{g}_{\mu_{0}}$-valued two-form on the reduced space $P_{\mu_{0}}$.

Theorem C of [3] shows that all curvature information on $\alpha_{\mu_{0}}$ is encoded in (i) the connection $D$ on the symplectic stratification of $P / G$ corresponding to the reconstruction connection $A$, together with (ii) the Poisson structure on $P / G$. 
The connection $\mathrm{D}$ allows to "assemble" the reduced symplectic structures $\omega_{\wedge}\left(\Lambda \subset \mathrm{P} / \mathrm{G}\right.$ a symplectic leaf) into a single two-form $\omega_{\mathrm{D}}$ on $\mathrm{P} / \mathrm{G}$ by decreeing that

$$
\omega_{\mathrm{D}}(u, v) \equiv \omega_{\wedge}\left(p_{\mathrm{D}} u, p_{\mathrm{D}} v\right), \quad u, v \in \mathrm{T}(\mathrm{P} / \mathrm{G}),
$$

where $\Lambda$ denotes the leaf to which the common base point of $u$ and $v$ belongs, and where $p_{D}: T(P / G) \rightarrow E$ denotes the projection along $D$ onto the characteristic distribution $\mathrm{E}$.

We remark that in general $\omega_{D}$ need not be smooth, but if $\mathrm{P}_{\mu_{0}}$ is a nondegenerate symplectic leaf, then $\omega_{D}$ is smooth wherever $D$ is of constant rank and smooth. Then, Corollary 4.5 of [3] states that assuming $G_{\mu_{0}}$ is Abelian and $\omega_{D}$ is smooth in a neighborhood of $\mathrm{P}_{\mu_{0}}$, the geometric phase is given by

$$
g_{\text {geom }}=\exp \int_{\Sigma} D_{\mu_{0}} \omega_{D} \text {. }
$$

\section{Connections on various bundles for Abelian groups}

In this section, the relation between connections on Poisson and symplectic bundles is analyzed. This establishes the validity of the application of results in [3] to our settings in the case of Abelian groups G, so that the metric orthogonal spaces to the group orbit in the whole tangent $\mathrm{T}_{x} \mathrm{P}$ as well as within the kernel $\operatorname{Ker} T J(x) \subset T_{\chi} P$ both constitute valid horizontal spaces for Poisson and symplectic bundles, respectively.

In Section 3.2, the formalism of dual pairs is introduced into the picture. The symplectic leaf correspondence theorem brings insight into the structure of various bundles and relates the corresponding connections. For the Abelian case, it gives a new interpretation of the connection on symplectic stratification $D$ as a connection on the bundle $j: P / G \rightarrow U \subset \mathfrak{g}^{*}$ of symplectic leaves over the dual of the Lie algebra (see Section 3.2).

\subsection{Connections on Poisson and symplectic bundles}

Consider the relation between a connection on the Poisson reduction bundle $\mathrm{P} \rightarrow \mathrm{P} / \mathrm{G}$ and connections on each of the symplectic Marsden-Weinstein reduction bundles $\mathrm{J}^{-1}(\mu) \rightarrow \mathrm{J}^{-1}(\mu) / \mathrm{G}_{\mu}$ for different $\mu \in \mathfrak{g}^{*}$. This relation can be easily established in the case of an Abelian group $G$ when $G_{\mu} \equiv G$ and both bundles have similar fibers.

Recall that a connection on the bundle $\mathrm{P} \rightarrow \mathrm{P} / \mathrm{G}$ is a Lie algebra valued one-form $\mathcal{A}$ on $\mathrm{P}$ that is $\mathrm{G}$-equivariant $\mathrm{g} \cdot \mathcal{A}=\operatorname{Ad}_{\mathrm{g}} \cdot \mathcal{A}$ and satisfies $\mathcal{A}\left(\xi_{\mathrm{p}}\right)=$ $\xi \forall \xi \in \mathfrak{g}$. The corresponding horizontal space is defined by hor $=\operatorname{Ker} \mathcal{A}$. The following theorem then holds.

Theorem 3.1. For the case of an Abelian group G, a connection $\mathcal{A}$ on the Poisson bundle induces connections $\alpha_{\mu}$ on symplectic Marsden-Weinstein 
bundles for regular momentum values $\mu$. In particular, it defines a reconstruction connection A on P. Moreover, the connections on the symplectic stratification of $\mathrm{P} / \mathrm{G}$ corresponding to $\mathrm{A}$ and to $\mathcal{A}$ coincide, that is,

$$
\widehat{A}=\mathrm{D}=\widehat{\mathcal{A}} .
$$

Proof. Choose a regular value $\mu \in \mathfrak{g}^{*}$ such that the symplectic reduction at $\mu$ is defined. Define induced horizontal and vertical spaces at $x \in J^{-1}(\mu)$ by the intersections with KerTJ:

$$
\operatorname{hor}_{\mu}=\text { hor } \cap \operatorname{KerTJ}, \quad \operatorname{ver}_{\mu}=\operatorname{ver} \cap \operatorname{KerTJ} .
$$

By definition, hor $_{\mu} \cap \operatorname{ver}_{\mu}=0$. As $G$ is Abelian, $G_{\mu}=G$, and $\operatorname{Ker} T \pi \subset$ $(\operatorname{Ker} T \pi)^{\omega}=\operatorname{KerTJ}$, so that $\operatorname{ver}_{\mu}=\operatorname{Ker} T \pi$. Using the following set-theoretical identity $(A+B) \cap C=A+B \cap C$ if $A \subset C$, we obtain

$$
\begin{aligned}
\operatorname{Ker} T J & =(\operatorname{Ker} T \pi+\text { hor }) \cap \operatorname{Ker} T J \\
& =\operatorname{Ker} T \pi+\text { hor } \cap \operatorname{KerTJ} \\
& =\operatorname{ver}_{\mu}+\text { hor }_{\mu} .
\end{aligned}
$$

Hence, $\operatorname{KerTJ}=\operatorname{ver}_{\mu} \oplus$ hor $_{\mu}$. The corresponding connection one-form $\alpha_{\mu}$ is defined by the horizontal space via $\operatorname{Ker} \alpha_{\mu}=$ hor $_{\mu}$. The collection of these $\alpha_{\mu}$ then define a reconstruction connection $A$ as defined in Section 2. It is G-invariant because $\mathcal{A}$ is $\mathrm{G}$-invariant for Abelian groups.

Finally, for the connections on the symplectic stratification of $\mathrm{P} / \mathrm{G}$ determined by connections on Poisson and symplectic bundles, that is, by $\mathcal{A}$ and $A$, respectively, we have at $y=\pi(x)$

$$
\begin{aligned}
\mathrm{D}^{\prime}(\mathrm{y}) & \equiv \widehat{\mathcal{A}}(\mathrm{x})=\mathrm{T} \pi\left(\operatorname{hor}^{\omega}(\mathrm{x})\right), \\
\mathrm{D}(\mathrm{y}) & \equiv \widehat{\mathcal{A}}(\mathrm{x})=\mathrm{T} \pi\left(\left(\operatorname{hor}_{\mu}\right)^{\omega}\right)=\mathrm{T} \pi\left((\operatorname{hor} \cap \operatorname{KerTJ})^{\omega}\right) \\
& =\mathrm{T} \pi\left(\left(\operatorname{hor}^{\omega}{ }^{\omega}+(\operatorname{Ker} \mathrm{TJ})^{\omega}\right)=\mathrm{T} \pi\left((\operatorname{hor})^{\omega}+\operatorname{Ker} \mathrm{T} \pi\right)=\mathrm{T} \pi\left(\operatorname{hor}^{\omega}(x)\right),\right.
\end{aligned}
$$

where $x \in \mathrm{J}^{-1}(\mu)$ with $y=\pi(x)$ and we have used that $(\operatorname{KerTJ})^{\omega}=\operatorname{Ker} \mathrm{T} \pi$. Comparing the last two expressions we conclude that $\mathrm{D}=\mathrm{D}^{\prime}$.

This result enables us to go back and forth between connections on Poisson and symplectic bundles for Abelian groups; in particular, it will let us apply results of [3] for the reconstruction phases and use the abstract mechanical connection (defined in Section 4) as a reconstruction connection.

\subsection{Connections on dual pairs}

Recall the notion of dual pairs introduced by Weinstein [13]. Consider a symplectic manifold $(P, \omega)$, Poisson manifolds $Q_{1}, Q_{2}$, and Poisson maps $\rho_{i}$ : 
$P \rightarrow Q_{i}, i=1,2$. If for almost all $x \in P,\left(\operatorname{Ker} T \rho_{1}(x)\right)^{\omega}=\operatorname{Ker} T \rho_{2}(x)$, the

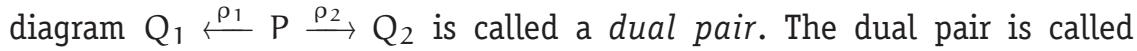

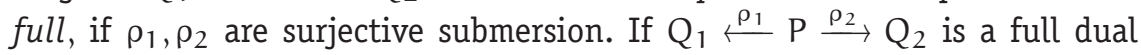
pair, then the spaces of Casimir functions on $\mathrm{Q}_{1}$ and $\mathrm{Q}_{2}$ are in bijective correspondence, that is, $\operatorname{Cas}\left(\mathrm{Q}_{1}\right) \circ \rho_{1}=\operatorname{Cas}\left(\mathrm{Q}_{2}\right) \circ \rho_{2}$ (Weinstein [13]).

It was shown in Adam and Ratiu [1] that for a symplectic manifold $(\mathrm{P}, \omega)$ with a Hamiltonian action of a Lie group $G$ having an equivariant momentum map $\mathrm{J}: \mathrm{P} \rightarrow \mathrm{U} \subset \mathfrak{g}^{*}, \mathrm{U} \equiv \mathrm{J}(\mathrm{P})$, such that $\pi: \mathrm{P} \rightarrow \mathrm{P} / \mathrm{G}$ and $\mathrm{J}$ are surjective submersion, $\mathrm{P} / \mathrm{G} \stackrel{\pi}{\leftarrow} \mathrm{P} \stackrel{\mathrm{I}}{\rightarrow} \mathrm{U}$ is a full dual pair. The Poisson reduced space $P / G$, being a base of a principle G-bundle, is itself foliated by symplectic leaves $\Sigma_{y}$ through points $y \in P / G$. We denote the space of symplectic leaves by $\mathcal{S}$. With the proper connectedness assumptions, these leaves are precisely the symplectic reduced spaces $P_{\mu}=J^{-1}(\mu) / G_{\mu}$ (note that $G_{\mu}$ can be different for different values of $\mu$ ).

On the other hand, $\mathrm{P}$ is foliated by the level sets of the momentum map $J^{-1}(\mu)$, for different $\mu \in \mathfrak{g}^{*}$, with the dual of the Lie algebra itself being a foliation by coadjoint orbits $\mathcal{O}_{\mu}$ through $\mu$. It follows from the symplectic leaf correspondence theorem [13] that, under the assumptions in the previous paragraph, the base space of this foliation is in one-to-one correspondence with $\mathcal{S}$, the space of symplectic leaves of the Poisson reduced space P/G. A natural one-to-one correspondence between the symplectic leaves in each leg of a dual pair has been described in Weinstein [13], together with a sketch of the proof. Here, we state the symplectic leaf correspondence theorem and refer for a detailed and comprehensive proof to Blaom [4].

Theorem 3.2. Let $\mathrm{P}$ be a symplectic manifold and $\mathrm{Q}_{1} \stackrel{\rho_{1}}{\longleftarrow} \mathrm{P} \stackrel{\rho_{2}}{\longrightarrow} \mathrm{Q}_{2}$ a full dual pair. Assume that each leg $\rho_{j}: \mathrm{P} \rightarrow \mathrm{Q}_{j}, j=1,2$ satisfies the property that pre-images of connected sets are connected. Let $\mathcal{F}_{j}$ denote the set of symplectic leaves in $\mathrm{Q}_{j}$. Then, under the assumptions outlined in Section 3.2 above, there exists a bijection $\mathcal{F}_{1} \rightarrow \mathcal{F}_{2}$ given by

$$
\Sigma_{1} \longmapsto \rho_{2}\left(\rho_{1}^{-1}\left(\Sigma_{1}\right)\right)
$$

having inverse

$$
\Sigma_{2} \longmapsto \rho_{1}\left(\rho_{2}^{-1}\left(\Sigma_{2}\right)\right) .
$$

This theorem enables us to define a leaf-to-leaf bijection that maps symplectic leaves $\Sigma_{y}$ (which are diffeomorphic to symplectic reduced spaces $\mathrm{P}_{\mu}$ ) to coadjoint orbits $\mathcal{O}_{\mu}$ in the dual of the Lie algebra, $\mu=J(x)$. Yet another realization of the symplectic leaves $\Sigma_{y}$ is given by the orbit reduction theorem [10] which establishes one-to-one correspondence between orbit reduced spaces $P_{\mathcal{O}_{\mu}}=J^{-1}\left(\mathcal{O}_{\mu}\right) / G$ and symplectic reduced spaces $P_{\mu}=J^{-1}(\mu) / G_{\mu}$.

In the case of an Abelian group $\mathrm{G}$, the coadjoint orbits are trivial, that is, $\mathcal{O}_{\mu}=\{\mu\}$ and $G_{\mu}=G$, so that $P_{\mathcal{O}_{\mu}} \equiv P_{\mu}$ and $\mathcal{S} \cong \mathfrak{g}^{*}$. It follows then from the 


\section{Abstract mechanical connection and Abelian reconstruction ...}

reduction lemma (cf. [10]) that G-orbits of any point $x \in P$ are isotropic, that is, $T_{x}(G \cdot x) \subset\left(T_{x}(G \cdot x)\right)^{\omega}$ or, equivalently, $\operatorname{Ker} T_{x} \pi \subset\left(\operatorname{Ker} T_{x} \pi\right)^{\omega}$. Moreover, the bijection $\mathcal{F}_{1} \rightarrow \mathcal{F}_{2}$ becomes a well-defined map of the manifolds, $j: P / G \rightarrow U$ which can be obtained through factoring the momentum map. Indeed, the equivariance of the momentum map $\mathrm{J}: \mathrm{P} \rightarrow \mathrm{U} \subset \mathfrak{g}^{*}$ amounts in the Abelian case to invariance. It therefore factors through $\pi: \mathrm{P} \rightarrow \mathrm{P} / \mathrm{G}$, delivering a map $\mathrm{j}: \mathrm{P} / \mathrm{G} \rightarrow \mathrm{U}$ making the diagram in Figure 3.1 commute.

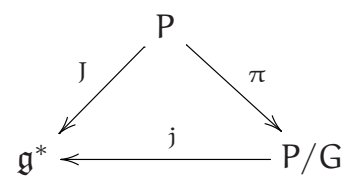

Figure 3.1. The momentum map J factors through delivering a map $j: P / G \rightarrow$ $\mathrm{U} \subset \mathfrak{g}^{*}$.

The map $j$ is a submersion since $J$ is a submersion (under our hypothesis of a free action). Since the coadjoint orbits are points, the symplectic leaves in $P / G$ are simply the fibers of $j$, that is, $P_{\mu}=j^{-1}(\mu), \mu \in U$.

Thus, with this interpretation, the connection on the symplectic stratification $\mathrm{D}$ can be thought of as an (Ehresmann) connection on the bundle $j: P / G \rightarrow U \subset \mathfrak{g}^{*}$. Theorem 2.1 of [3] as well as the results of Section 3 establish a relation between the connections $A$ and $D$ on the bundles $\pi: P \rightarrow P / G$ and $\mathrm{j}: \mathrm{P} / \mathrm{G} \rightarrow \mathrm{U} \subset \mathfrak{g}^{*}$, respectively.

Finally, the tangent map $\mathrm{Tj}$ delivers the isomorphism of Proposition 2.5, where now $L$ does not depend explicitly on $\mu$ as $G$ is Abelian; that is, $\mathfrak{g}_{\mathfrak{\mu}}^{*}=\mathfrak{g}^{*}$, $p_{\mu} \equiv \mathrm{Id}$, and the dependence on $\mu$ enters only through $\mu=\mathfrak{j}(\mathrm{y})$.

Lemma 3.3. Let $\mathrm{L}(\mathrm{D}, \mathrm{y}): \mathfrak{g}^{*} \rightarrow \mathrm{D}(\mathrm{y})$ be defined by (2.6), then its inverse is given by the tangent map $\mathrm{Tj}$ restricted to the distribution $\mathrm{D}$

$$
\mathrm{L}^{-1}=\operatorname{forg}\left(\left.\mathrm{Tj}\right|_{\mathrm{D}}\right): \mathrm{D} \longrightarrow \mathfrak{g}^{*},
$$

where the map forg: TU $\rightarrow \mathfrak{g}^{*}$ denotes the map that "forgets base point."

Proof. The proof readily follows from the fact that the momentum map factors through the quotient map, so that $T J=T j \circ T \pi$, and the definition of the map $L$ for any $y \in P_{\mu}$ given by (2.6), where $w$ is any vector in $T_{x} P$ that satisfies $\mathrm{T} \pi \cdot w=v$, with $v \in \mathrm{D}(\mathrm{y})$ and $x \in \mathrm{J}^{-1}(\mu) \cap \pi^{-1}(\mathrm{x})$ :

$$
\begin{aligned}
v \longmapsto \mathrm{L}^{-1}(\mathrm{D}, \mathrm{y}) \cdot v & \equiv p_{\mu}(\operatorname{forg}(\mathrm{TJ} \cdot w)) \\
& =\text { forg }(\mathrm{Tj} \circ \mathrm{T} \pi \cdot w)=\text { forg }(\mathrm{Tj} \cdot v) .
\end{aligned}
$$




\section{Abstract mechanical connection}

Let $P$ be an almost Kähler manifold with a complex structure $\mathcal{J}: T_{x} P \rightarrow T_{x} P$, such that $\mathcal{J}^{2}=-1$, a symplectic form $\omega$ and a $\mathcal{J}$-invariant Riemannian metric $\mathfrak{s}$ with the standard relation between these structures, (1.1),

$$
\omega(v, w)=\mathfrak{s}(\mathcal{J} v, w) \quad \forall v, w \in \mathrm{T}_{x} \mathrm{P} .
$$

Let a Lie group $G$ act on P freely and properly (see [12] for some interesting results on how to relax the regularity conditions) by isometries of the almost Kähler structure, that is, it preserves Riemannian, symplectic, and almost complex forms. The quotient manifold then has a unique Poisson structure such that the canonical projection $\pi: \mathrm{P} \rightarrow \mathrm{P} / \mathrm{G}$ is a Poisson map. Assume that the $\mathrm{G}$ action admits an equivariant momentum map $\mathrm{J}$ and that $\mathrm{P} / \mathrm{G} \stackrel{\pi}{\longleftarrow}$ $\mathrm{P} \stackrel{\mathrm{J}}{\longrightarrow} \mathrm{U} \subset \mathfrak{g}^{*}$ is a full dual pair, that is, $\pi$ and J are surjective submersions. Though we are not interested here in the results for Kähler reduction (The reader is referred to [5, 12], for example, for Marsden-Weinstein reduction on Kähler manifolds.) we notice that the almost complex structure can be dropped to the quotient space $\mathrm{P} / \mathrm{G}$. We keep the same notation for the reduced object but write $\mathcal{J}(y)$, where $y=\pi(x)$, to indicate that it can be computed at any $x \in \pi^{-1}(y)$.

\subsection{Main constructions}

Definition 4.1. The abstract locked inertia tensor $\mathbb{I}(x): \mathfrak{g} \rightarrow \mathfrak{g}^{*}, \forall x \in P$, is defined by the following expression:

$$
\langle\mathbb{I}(x) \cdot \xi, \eta\rangle=\mathfrak{s}\left(\xi_{\mathrm{P}}(x), \eta_{\mathrm{P}}(x)\right)
$$

for any Lie algebra elements $\xi, \eta \in \mathfrak{g}$, where $\xi_{\mathrm{P}}, \eta_{\mathrm{P}}$ are the corresponding infinitesimal generators, that is, vector fields on $\mathrm{P}$.

The abstract locked inertia tensor is, obviously, an isomorphism for any $x \in \mathrm{P}$ for which the group action is free. For a general Lie group, it is Gequivariant in the sense of a map $\mathbb{I}: \mathrm{P} \rightarrow \mathrm{L}\left(\mathfrak{g}, \mathfrak{g}^{*}\right)$, namely

$$
\mathbb{I}(\mathrm{g} \cdot \mathrm{x}) \cdot \operatorname{Ad}_{\mathrm{g}} \xi=\operatorname{Ad}_{\mathrm{g}^{-1}}^{*} \mathbb{I}(\mathrm{x}) \cdot \xi .
$$

For an Abelian group, the abstract locked inertia tensor is, in fact, Ginvariant and, hence, can be dropped to the quotient $P / G$. We use the same notation for the reduced object but write $\mathbb{I}(y)$, where $y=\pi(x)$, to indicate that it can be computed at any $x \in \pi^{-1}(y)$.

Definition 4.2. For any choice of a principle connection on $P / G$, define the induced metric $\mathfrak{s}^{\prime}$ on $P / G$ in the following way. Let $a, b \in T_{y}(P / G)$ and let $\tilde{a}, \tilde{b}$ be their corresponding pre-images in the horizontal subspace, that is, 
14 Abstract mechanical connection and Abelian reconstruction ...

$\tilde{a}, \tilde{b} \in \operatorname{hor}(x), \pi(\tilde{a})=a$, and $\pi(\tilde{a})=b$, where $x \in \pi^{-1}(y)$. As the metric is G-invariant we can define $\mathfrak{s}^{\prime}(a, b)=\mathfrak{s}_{x}(\tilde{a}, \tilde{b})$, for any $x \in \pi^{-1}(y)$.

Definition 4.3. The abstract mechanical connection on the principle Gbundle $\mathrm{P} \rightarrow \mathrm{P} / \mathrm{G}$ is defined by specifying a horizontal space within $\mathrm{T}_{\chi} \mathrm{P}$ at each point $x \in P$ to be metric-orthogonal to the tangent to the group orbits

$$
\operatorname{hor}(x)=\left\{v \in \mathrm{T}_{\mathbf{x}} \mathrm{P} \mid \mathfrak{s}\left(v, \xi_{\mathrm{P}}(x)\right)=0 \forall \xi \in \mathfrak{g}\right\} .
$$

The connection one-form $\mathcal{A}$ is determined by $\operatorname{Ker} \mathcal{A}(x)=\operatorname{hor}(x)$; an explicit expression for it is given by the following theorem.

Theorem 4.4. Abstract mechanical connection on an almost Kähler manifold is given by

$$
\mathcal{A}(x) \cdot w=\mathbb{I}^{-1}(x) \cdot \mathfrak{s}\left(\omega^{\#}(\mathrm{dJ}(\mathrm{x})), w\right) \quad \forall w \in \mathrm{T}_{\mathrm{x}} \mathrm{P} .
$$

Proof. For any tangent vector $w \in \mathrm{T}_{\chi} \mathrm{P}$ and any Lie algebra element $\eta \in \mathfrak{g}$ :

$$
\mathfrak{s}\left(\mathcal{w}, \eta_{\mathrm{P}}\right)=\mathfrak{s}\left(w_{v}+w_{\mathrm{h}}, \eta_{\mathrm{P}}\right)=\mathfrak{s}\left(\mathcal{w}_{v}, \eta_{\mathrm{P}}\right)=\mathfrak{s}\left(\xi_{\mathrm{P}}^{w}, \eta_{\mathrm{P}}\right)=\left\langle\mathbb{I}(x) \xi^{w}, \eta\right\rangle
$$

where $\mathcal{w}_{v}=\xi_{\mathrm{P}}^{\mathcal{w}}$ for some $\xi^{w} \in \mathfrak{g}$ is a vertical (fiber) component, $w_{\mathrm{h}}$ is a horizontal component, and $\mathfrak{s}\left(\mathcal{w}_{\mathrm{h}}, \eta_{\mathrm{P}}\right)=0$ by definition.

By definition of the momentum map $\eta_{P}=\omega^{\#}(d\langle J(x), \eta\rangle)$, so that

$$
\mathfrak{s}\left(\mathcal{w}, \eta_{\mathrm{P}}\right)=\mathfrak{s}\left(\mathcal{w}, \omega^{\#}(\mathrm{~d}\langle\mathrm{~J}(\mathrm{x}), \eta\rangle)\right)=\left\langle\mathfrak{s}\left(\omega^{\#}(\mathrm{dJ}), w\right), \eta\right\rangle,
$$

where $\mathrm{dJ}$ is thought of as a $\mathfrak{g}^{*}$-valued one-form on $\mathrm{P}$, and we have used the fact that the pairing between $\mathfrak{g}$ and its dual is independent of $x \in P$. Thus,

$$
\left\langle\mathbb{I}(x) \xi^{w}, \eta\right\rangle=\left\langle\mathfrak{s}\left(\omega^{\#}(\mathrm{dJ}), w\right), \eta\right\rangle,
$$

and the result follows from the nondegeneracy of the pairing.

To verify that $\mathcal{A}$ indeed defines a connection, we check that it satisfies $\mathcal{A}\left(\xi_{\mathrm{P}}(x)\right)=\xi \forall \xi \in \mathfrak{g}$ and is G-equivariant. Consider the pairing of $\mathbb{I}(x) \cdot \mathcal{A}\left(\xi_{\mathrm{P}}(x)\right)$ with an arbitrary element from the Lie algebra $\eta \in \mathfrak{g}$ and use Definitions 4.1, 4.2, and 4.3 of the connection and the abstract locked inertia tensor:

$$
\begin{aligned}
\left\langle\mathbb{I}(x) \cdot \mathcal{A}\left(\xi_{P}(x)\right), \eta\right\rangle & =\left\langle\mathfrak{s}\left(\omega^{\#}(\mathrm{dJ}(x)), \xi_{P}(x)\right), \eta\right\rangle \\
& =\mathfrak{s}\left(\xi_{P}, \eta_{P}\right)=\langle\mathbb{I}(x) \xi, \eta\rangle .
\end{aligned}
$$

From the nondegeneracy of the pairing, it follows that $\mathcal{A}\left(\xi_{\mathrm{P}}(x)\right)=\xi$. The G-equivariance means that $\Phi_{\mathrm{g}}^{*} \mathcal{A}=\operatorname{Ad}_{\mathrm{g}} \mathcal{A}$ and follows from equivariance of the momentum map and equivariance of the abstract locked inertia tensor in the sense of a map $\mathbb{I}: \mathrm{P} \rightarrow \mathrm{L}\left(\mathfrak{g}, \mathfrak{g}^{*}\right)$ (see (4.3)). 
Corollary 4.5. The connection one-form can be written as follow:

$$
\mathcal{A}(\mathrm{x}) \cdot \mathfrak{w}=\mathbb{I}^{-1}(\mathrm{x}) \cdot \text { forg }(\mathrm{TJ}(\mathrm{x})(\mathcal{J w})) \quad \forall w \in \mathrm{T}_{\mathrm{x}} \mathrm{P} .
$$

Then,

$$
\operatorname{hor}(x)=\operatorname{Ker}(\mathrm{TJ}(x) \circ \mathcal{J}) \text {. }
$$

Proof. Using (1.1), $\mathrm{J}^{2}=-1$ and omitting $x$ for simplicity, we have $\forall w \in \mathrm{T}_{\chi} \mathrm{P}$

$$
\begin{aligned}
\mathcal{A} \cdot w & =\mathbb{I}^{-1} \cdot \mathfrak{s}\left(\omega^{\#}(\mathrm{dJ}),-\mathcal{J}^{2} w\right) \\
& =\mathbb{I}^{-1} \cdot \omega\left(\omega^{\#}(\mathrm{dJ}), \partial w\right) \\
& =\mathbb{I}^{-1} \cdot \operatorname{forg}(\mathrm{TJ}(\partial w))
\end{aligned}
$$

where for the last equality we used the definition of a symplectic form and considered the one-form $d_{x} J$ as a tangent map forg $\circ T$ J acting on vectors in $T_{x} P$.

Lemma 4.6. For the choice of the abstract mechanical connection $\mathcal{A}$ on $\mathrm{P}$ with hor $=(\operatorname{Ker} T \pi)^{\perp}$, the following holds:

$$
\operatorname{hor}^{\omega}=(\operatorname{Ker} \mathrm{TJ})^{\perp}=\mathcal{J}(\operatorname{Ker} \mathrm{T} \pi)
$$

Proof. The proof follows readily from (4.11) of Corollary 4.5 and the $\omega$ orthogonality of KerTJ and $\operatorname{Ker} T \pi$ :

$$
\begin{aligned}
\operatorname{hor}^{\omega}=(\operatorname{Ker}(\mathrm{TJ} \circ \mathcal{J}))^{\omega} & =(\mathcal{J}(\operatorname{Ker} \mathrm{TJ}))^{\omega}=\left(\left((\operatorname{Ker} \mathrm{TJ})^{\perp}\right)^{\omega}\right)^{\omega} \\
& =(\operatorname{Ker} \mathrm{TJ})^{\perp}=\left((\operatorname{Ker} \mathrm{T} \pi)^{\omega}\right)^{\perp}=\mathcal{J}(\operatorname{Ker}(\mathrm{TJ})),
\end{aligned}
$$

where we used that $\left((W)^{\omega}\right)^{\perp}=\mathcal{J}(W)$ for a subspace $W \in T_{x} P$.

Below we present two alternative proofs of this lemma which provide an interesting insight into the issue; these proofs can be skipped on the first reading.

Alternative proof. By definition, $w \in \operatorname{hor}^{\omega}(x)$ if and only if

$$
\omega(v, w)=\mathfrak{s}(v, \mathcal{J w})=0 \quad \forall v \in \operatorname{hor}(x)
$$

Thus, $w \in \operatorname{hor}^{\omega}(x) \Leftrightarrow \mathcal{J w} \in(\operatorname{hor}(x))^{\perp}$, or

$$
w \in\left(\operatorname{hor}^{\omega}(x)\right)^{\perp} \Longleftrightarrow \mathcal{J} \in \operatorname{hor}(x)=(\operatorname{Ker} T \pi(x))^{\perp} .
$$

On the other hand, $u \in(\operatorname{Ker} T \pi(x))^{\omega}=\operatorname{Ker} T J(x)$ if and only if

$$
\omega\left(u, \xi_{P}(x)\right)=\mathfrak{s}\left(\xi_{P}(x), \mathcal{J u}\right)=0 \quad \forall \xi \in \mathfrak{g} .
$$


16 Abstract mechanical connection and Abelian reconstruction ...

Thus,

$$
u \in \operatorname{KerTJ}(x) \Longleftrightarrow \mathcal{J u} \in\left(\mathrm{T}_{x}(\mathrm{G} \cdot \mathrm{x})\right)^{\perp} \equiv(\operatorname{Ker} \mathrm{T} \pi(x))^{\perp} .
$$

Comparing conditions for $u$ and $w$, we conclude that $\operatorname{hor}^{\omega}(x)=$ $(\operatorname{Ker} T J(x))^{\perp}$. Then, (4.18) follows from $\operatorname{Ker} T J=(\operatorname{Ker} T \pi)^{\omega}$ and $\left((\operatorname{Ker} T \pi)^{\omega}\right)^{\perp}$ $=\mathcal{J}(\operatorname{Ker} \mathrm{T} \pi)$.

Alternative proof. First notice that

$$
\operatorname{hor}^{\omega}=\left((\operatorname{Ker} T \pi)^{\perp}\right)^{\omega}=\left((\operatorname{Ker} T \pi)^{\omega}\right)^{\perp}=(\operatorname{Ker} \mathrm{TJ})^{\perp} .
$$

The last equality in (4.19) follows from the following argument. Let $A \subset T_{x} P$, then $a \in A^{\perp} \Leftrightarrow \mathfrak{s}(a, b)=0 \forall b \in A$. Similarly, $c \in\left(A^{\perp}\right)^{\omega} \Leftrightarrow \omega(c, a)=0 \forall a \in A^{\perp}$. But $0=\omega(c, a)=\mathfrak{s}(\mathcal{J c}, a) \forall a \in A^{\perp}$ implies that

$$
c \in\left(A^{\perp}\right)^{\omega} \Longleftrightarrow \mathcal{c} \in\left(A^{\perp}\right)^{\perp} \equiv A .
$$

This is equivalent to $c \in \mathcal{J}(A) \Leftrightarrow c \in\left(A^{\perp}\right)^{\omega}$, so that $\left(A^{\perp}\right)^{\omega}=\mathcal{J}(A)$ and $\left((\operatorname{Ker} T \pi)^{\perp}\right)^{\omega}=\mathcal{J}(\operatorname{Ker} T \pi)$.

Define for any point $v \in \mathfrak{g}^{*}$ a one-form $\mathcal{A}_{v}(x)=\langle v, \mathcal{A}(x)\rangle$ on $\mathrm{P}$.

Lemma 4.7. Identifying vectors and one-forms on $\mathrm{P}$ via Riemannian metric

$$
\left(\mathcal{A}_{v}(x)\right)^{\#}=\left(\mathbb{I}^{-1}(x) \cdot v\right)_{\mathrm{P}} .
$$

Proof. Using (4.5) we obtain $\forall w \in \mathrm{T}_{x} \mathrm{P}$

$$
\begin{aligned}
\mathcal{A}_{v} \cdot w & =\left\langle v, \mathbb{I}^{-1} \cdot \mathfrak{s}\left(\omega^{\#}(\mathrm{dJ}), w\right)\right\rangle \\
& =\mathfrak{s}\left(\omega^{\#}\left(\mathrm{~d}\left\langle J, \mathbb{I}^{-1} v\right\rangle\right), w\right)=\mathfrak{s}\left(\left(\mathbb{I}^{-1} v\right)_{\mathrm{P}}, w\right) .
\end{aligned}
$$

\subsection{Abelian groups and reconstruction phases}

In the rest of this section we assume that the Lie group $\mathrm{G}$ is Abelian. A simple corollary of Theorem 3.1 implies that metric orthogonal horizontal spaces on the Poisson bundle $\mathrm{P} \rightarrow \mathrm{P} / \mathrm{G}$ induce metric orthogonal horizontal spaces on symplectic bundles $\mathrm{J}^{-1}(\mu) \rightarrow \mathrm{P}_{\mu}$ for regular $\mu$. Hence, by analogy, the reconstruction connection $A$ corresponding to $\mathcal{A}$ by means of Theorem 3.1 can be called an abstract mechanical reconstruction connection. The same theorem gives also the corresponding connection on the symplectic stratification $\mathrm{D}=\widehat{A}$ by specifying its horizontal spaces to be $T \pi$ (hor $^{\omega}$ ). The following results significantly simplify explicit computations of these spaces, that is, the distribution D. 
Theorem 4.8. For the choice of the abstract mechanical connection $\mathcal{A}$ on $\mathrm{P}$, the distribution $\mathrm{D}$, which corresponds to the connection on the symplectic stratification $\mathrm{j}: \mathrm{P} / \mathrm{G} \rightarrow \mathfrak{g}^{*}$, is metric orthogonal to the characteristic distribution $\mathrm{E}$ in the metric $\mathfrak{s}^{\prime}$ induced on the quotient $\mathrm{P} / \mathrm{G}$. Moreover, the distribution $\mathrm{D}$ can be explicitly constructed using the infinitesimal generator vector fields $\xi_{\mathrm{p}}$ according to the following expression:

$$
\mathrm{D}(\mathrm{y})=\mathrm{T} \pi(\mathcal{J}(\operatorname{Ker} \mathrm{T} \pi(x))),
$$

where $x \in \pi^{-1}(y)$ and $\operatorname{Ker} T \pi(x)=\operatorname{span}\left\{\xi_{p}(x)\right\}$.

Proof. Consider any vectors $v \in \mathrm{D}(y)$ and $w \in \mathrm{E}(\mathrm{y}) \equiv \mathrm{T}_{y} \Sigma_{y}$. By definition of the induced metric $\mathfrak{s}^{\prime}(v, w)=\mathfrak{s}(\tilde{v}, \tilde{w})$, where $\tilde{v}, \tilde{w} \in \operatorname{hor}(x)$ are horizontal components of the pre-images: $\mathrm{T} \pi(\tilde{v})=v, \mathrm{~T} \pi(\tilde{w})=\mathcal{w}$, and $x \in \pi^{-1}(y)$.

From $\mathrm{T} \pi(\tilde{v})=v \in \mathrm{T} \pi\left(\right.$ hor $\left.^{\omega}\right)$ it follows that $\tilde{v} \in$ hor $^{\omega}+\operatorname{Ker} T \pi$. But $\tilde{v} \in$ hor $\equiv(\operatorname{Ker} T \pi)^{\perp}$, so that by Lemma 4.6,

$$
\tilde{v} \in \operatorname{hor}^{\omega} \cap(\operatorname{Ker} T \pi)^{\perp} \equiv(\operatorname{KerTJ})^{\perp} \cap(\operatorname{Ker} T \pi)^{\perp} .
$$

For the vector $w \in \mathrm{E}(\mathrm{y})$ it holds $\mathrm{Tj}(w)=0$ and, hence, by the commutativity of the diagram in Figure 3.1, $\mathrm{TJ}(\tilde{\mathcal{w}})=0$ for any of its pre-images. In particular, for the horizontal pre-image $\tilde{w} \in$ hor we have

$$
\tilde{w} \in \operatorname{KerTJ} \cap(\operatorname{Ker} T \pi)^{\perp} .
$$

From the expressions for $\tilde{v}$ and $\tilde{w}$, it follows that $\mathfrak{s}^{\prime}(\nu, w)=\mathfrak{s}(\tilde{v}, \tilde{w})=0$.

Finally, (4.23) follows from $\mathrm{D}=\mathrm{T} \pi\left((\text { hor })^{\omega}\right)$ and Lemma 4.6.

\subsubsection{Transverse derivatives}

Here we give a new construction of the map L defined by Proposition 2.5 which is crucial for the definition of the transverse derivatives, and hence for the computation of the phases. Our construction is based on Lemma 3.3 and depends implicitly on the choice of the abstract mechanical connection.

Definition 4.9. For each point $y \in P / G$ define a map $N(D, y): \mathfrak{g} \rightarrow D(y)$ by

$$
\xi \longmapsto \mathrm{T} \pi\left(\mathcal{J}\left(\xi_{\mathrm{P}}(\mathrm{x})\right)\right),
$$

where $\xi$ is a Lie algebra element, $\xi_{\mathrm{P}}(x)$ is its corresponding infinitesimal generator at $x \in \pi^{-1}(y) \subset P$, and $\mathcal{J}$ is the almost complex structure on $P$.

From (4.26) it follows that $\mathrm{N}$ is a linear map as all maps used in its definition are linear. From the symplectic leaf correspondence theorem and the fact that $\mathrm{G}$ is Abelian and finite dimensional it follows that the dimension of 
18 Abstract mechanical connection and Abelian reconstruction ...

$\mathrm{D}(\mathrm{y})$ (which equals the co-dimension of the leaf $\Sigma_{y}$ ) equals the dimension of the algebra $\mathfrak{g}$. On the other hand,

$$
\operatorname{dim}\left(\operatorname{hor}^{\omega}(x)\right)=\operatorname{dim}(\mathcal{J}(\operatorname{Ker} \operatorname{T} \pi(x)))=\operatorname{dim}(\operatorname{Ker} \operatorname{T}(x))=\operatorname{dim} \mathfrak{g} .
$$

Hence, from the fact that $D=T \pi$ hor $^{\omega}$ ) the following lemma follows.

Lemma 4.10. For each $\mathrm{y} \in \mathrm{P} / \mathrm{G}$ the map $\mathrm{N}$ is an isomorphism between the Lie algebra $\mathfrak{g}$ and the transverse space $\mathrm{D}(\mathrm{y})$ defined at $\mathrm{y}$ by the distribution $\mathrm{D}$ on the symplectic stratification $\mathrm{j}: \mathrm{P} / \mathrm{G} \rightarrow \mathfrak{g}^{*}$.

Lemma 4.11. For an Abelian group G, the map L(D,y) defined in Proposition 2.5 is given by the following composition:

$$
\mathrm{L}(\mathrm{D}, \mathrm{y})=\mathrm{N}(\mathrm{D}, \mathrm{y}) \circ \mathbb{I}^{-1}(\mathrm{y}): \mathfrak{g}^{*} \longrightarrow \mathrm{D}(\mathrm{y}),
$$

where $\mathbb{I}$ is the abstract locked inertia tensor.

Proof. By the definition of the momentum map, $\mathrm{J}_{\xi} \equiv\langle\mathrm{J}, \xi\rangle$ is a Hamiltonian for the vector field $\xi_{P}$ of the infinitesimal transformations, that is, for any vector $u \in T_{x} P$

$$
\omega(x)\left(\xi_{P}, u\right)=d_{x} J_{\xi}(u) .
$$

The one-form $d_{x} J_{\xi}$ can be thought of as the tangent map TJ acting on vectors in $T_{x} P$ and paired with $\xi \in \mathfrak{g}$. Take $u$ to be $\mathcal{J}\left(\eta_{P}\right)$ for some infinitesimal generator $\eta_{P}$ corresponding to $\eta \in \mathfrak{g}$. Then,

$$
\begin{aligned}
\omega(x)\left(\xi_{P}, \mathcal{J}\left(\eta_{P}\right)\right) & =d_{x} J_{\xi}\left(\mathcal{J}\left(\eta_{P}\right)\right)=\left\langle d_{x} J\left(\mathcal{J}\left(\eta_{P}\right)\right), \xi\right\rangle \\
& =\left\langle\operatorname{forg}\left(T J\left(\mathcal{J}\left(\eta_{P}\right)\right)\right), \xi\right\rangle \\
& =\left\langle\operatorname{forg}\left(\operatorname{Tj} \circ T \pi\left(\mathcal{J}\left(\eta_{P}\right)\right)\right), \xi\right\rangle \\
& =\langle\operatorname{forg}(\operatorname{Tj} \circ N(\eta)), \xi\rangle,
\end{aligned}
$$

where we used the definition of the map $N$ given by (4.26).

On the other hand,

$$
\begin{aligned}
\omega(x)\left(\xi_{P}, \mathcal{J}\left(\eta_{P}\right)\right) & =-\omega(x)\left(\mathcal{J}\left(\eta_{P}\right), \xi_{P}\right)=\mathfrak{s}(x)\left(\eta_{P}, \xi_{P}\right) \\
& =\langle\mathbb{I}(x) \eta, \xi\rangle=\langle\mathbb{I}([x]) \eta, \xi\rangle=\langle\mathbb{I}(y) \eta, \xi\rangle .
\end{aligned}
$$

Alternatively, this expression can be obtained from Corollary 4.5 using an explicit form of the connection one-form given by (4.10).

From (4.30) and (4.31) and the nondegeneracy of the pairing we conclude that forg $(T j \circ N)=\mathbb{I}$, then from Lemma 3.3 it follows that

$$
L(D, y)=\text { forg }\left(\left.T j\right|_{D}\right)^{-1}=N(D, y) \circ \mathbb{I}^{-1}(y) .
$$

Notice that $\mathrm{L}$ is an isomorphism as both $\mathrm{N}$ and $\mathbb{I}$ are. 


\subsubsection{Dynamic phase}

Recall that according to (2.15), the infinitesimal dynamic phase is given by the transverse derivative of the reduced Hamiltonian, which we simplify using formula (4.32) for the map L.

Theorem 4.12. The v-component of the infinitesimal dynamic phase $\xi_{\text {dyn }}$, for any $v \in \mathfrak{g}^{*}$, can be expressed via the abstract locked inertia tensor and the almost complex structure according to

$$
\left\langle v, \xi_{\text {dyn }}(y)\right\rangle=\left\langle v, D_{\mu} h(y)\right\rangle=\operatorname{dh}\left(\operatorname{T} \pi\left(\mathcal{J}([x])\left(\left(\mathbb{I}^{-1}([x]) \cdot v\right)_{P}\right)\right)\right),
$$

where $x \in[x]=\pi^{-1}(y)$ and $\mu=j(y)$.

Proof. The proof is quite straightforward and relies on the constructions discussed in this section. Using the definition of the transverse derivative, Lemma 4.11 and G-invariance of the abstract locked inertia tensor, and the almost complex structure, we obtain

$$
\begin{aligned}
\left\langle v, \xi_{\text {dyn }}(y)\right\rangle & =\left\langle v, D_{\mu} h(y)\right\rangle=\operatorname{dh}(L(D, \mu, y) \cdot v) \\
& =\operatorname{dh}\left(N(D, y) \circ \mathbb{I}^{-1}(x) \cdot v\right) \\
& =\operatorname{dh}\left(T \pi\left(J(x)\left(\left(\mathbb{I}^{-1}(x) \cdot v\right)_{P}\right)\right)\right)
\end{aligned}
$$

where the last equality follows from (4.26).

As it was pointed out earlier, both $\mathcal{J}$ and $\mathbb{I}$ are G-invariant and, hence, can be dropped to the quotient $P / G$, so that (4.33) can be computed at any $x \in[x] \equiv y$.

Remark 4.13. Equation (4.33) is equivalent to

$$
\left\langle v, \xi_{\operatorname{dyn}}(y)\right\rangle=\operatorname{dH}\left(\mathcal{J}(x)\left(\left(\mathbb{I}^{-1}(x) \cdot v\right)_{P}\right)\right),
$$

where $x \in \pi^{-1}(y)$ and $\mu=j(y)$.

Notice that (4.33) does not depend on the choice of $x \in \pi^{-1}(y)$. This agrees with the general philosophy of [3] that all information about the phases is contained in the reduced quantities. Yet, for the explicit computations it might be convenient to work with the objects in the unreduced space. Alternatively, when one has a good model of the reduced space $P / G$, one can compute a basis $v_{k}$ of the distribution $D$ at any $y \in P / G$ using isomorphism $L$ and Lemma 4.11 corresponding to a basis $e_{i}$ of $\mathfrak{g}^{*}$. Then, for any $v=\sum v^{i} e_{i} \in \mathfrak{g}^{*}$, the corresponding $v$-component of the dynamic phase is given by the derivative of the reduced Hamiltonian in the direction $v=\sum v^{k} \boldsymbol{v}_{\mathrm{k}}$, that is, $\left\langle v, \xi_{\mathrm{dyn}}(y)\right\rangle=\operatorname{dh}\left(\sum v^{\mathrm{k}} \boldsymbol{v}_{\mathrm{k}}\right)$. 
Abstract mechanical connection and Abelian reconstruction ...

\subsubsection{Geometric phase}

Assuming the $\mu$-regularity of the distribution D (see Section 2), the geometric phase is given by the transverse derivative of the assembled reduced symplectic form $\omega_{\mathrm{D}}$ according to (2.17). In this section, we give an explicit construction of this form $\omega_{D}$ using the horizontal lifts with respect to the abstract mechanical connection $A$ and the unreduced symplectic form $\omega$. This allows us to circumvent explicit computations of the curvature of the connection one-form that is used in (2.16) and, in some cases, also the computations of the reduced symplectic form that is used in (2.17).

Definition 4.14. For an Abelian group G, define a closed "horizontal" twoform $\omega^{\prime}$ on $\mathrm{P} / \mathrm{G}$ according to

$$
\omega^{\prime}(y)(u, v):=\omega(x)(\tilde{u}, \tilde{v}) \quad \forall u, v \in T_{y}(P / G), y \in P_{\mu}
$$

where $\tilde{u}, \tilde{v} \in \mathcal{A}(x) \equiv \operatorname{hor}(x) \equiv(\operatorname{Ker} T \pi)^{\perp}$ with $\operatorname{T} \pi(\tilde{u})=u, \operatorname{T} \pi(\tilde{v})=v, x \in$ $\pi^{-1}(y)$.

From the G-invariance of the symplectic form $\omega$ as well as of the horizontal distribution $\mathcal{A}$ we conclude that $\omega^{\prime}$ is well defined.

Theorem 4.15. The two-form $\omega^{\prime}$ coincides with the assembled two-form $\omega_{\mathrm{D}}$ on $\mathrm{P} / \mathrm{G}$ :

$$
\omega^{\prime}=\omega_{\mathrm{D}}
$$

Proof. We start with the definition of the two-form $\omega^{\prime}$ Definition 4.14 of the two-form and shall demonstrate that the following three special cases hold for any $y \in P / G$ :

(1) $\left.\omega^{\prime}\right|_{E}=\omega_{\mu}$, here $E$ is the characteristic distribution and $\mu=j(y)$,

(2) $\left.\omega^{\prime}\right|_{D}=0$,

(3) $\omega^{\prime}(u, v)=0$ for any $u \in E(y) \equiv T_{y} P_{\mu}$ and $v \in D(y)$,

which all together prove the statement of the theorem, according to the definition of the assembled form (2.17).

(1) From the definition of the reduced symplectic form in the MarsdenWeinstein reduction it follows that

$$
\omega_{\mu}(y)(u, v)=\omega(x)(\breve{u}, \breve{v}),
$$

where $x \in \pi^{-1}(y) \cap J^{-1}(\mu)$ and $\breve{u}, \breve{v} \in A(x)$, that is, the pre-images lie in the horizontal space of the reconstruction connection $A$. Recall that in our case, A denotes the metric orthogonal to the group orbit within the kernel of TJ:

$$
\mathrm{A}(\mathrm{x})=\left(\mathrm{T}_{x}(\mathrm{G} \cdot \mathrm{x})\right)^{\perp} \cap \operatorname{KerTJ}(\mathrm{x})=(\operatorname{Ker} \mathrm{T} \pi(\mathrm{x}))^{\perp} \cap \operatorname{Ker} \mathrm{TJ}(\mathrm{x}) .
$$


From Lemma 2.3 and the fact that G-orbits of any point $x \in P$ are isotropic for an Abelian group, it follows that

$$
\pi^{*} \mathrm{E}=\operatorname{Ker} \mathrm{T} \pi+(\operatorname{Ker} \mathrm{T} \pi)^{\omega}=(\operatorname{Ker} \mathrm{T} \pi)^{\omega} .
$$

Hence, using the definition on the two-form $\omega^{\prime}$, Definition 4.14 of the twoform, for any vectors $u, v \in E=T_{y} P_{\mu}$ lying in the characteristic distribution at $y \in \mathrm{P}_{\mu}$, their pre-images $\tilde{u}, \tilde{v} \in \mathcal{A}(\mathrm{x})$ satisfy

$$
\tilde{u}, \tilde{v} \in \mathcal{A}(x) \cap(\operatorname{Ker} T \pi)^{\omega}=(\operatorname{Ker} T \pi)^{\perp} \cap \operatorname{Ker} T J=A(x),
$$

so that

$$
\omega^{\prime}(y)(u, v):=\omega(x)(\tilde{u}, \tilde{v})=\omega(x)(\breve{u}, \breve{v})=\omega_{\mu}(y)(u, v) .
$$

(2) Let $u, v \in D(y)$, then $\tilde{u}, \tilde{v} \in \pi^{*} D=A^{\omega}$, by the definition of the reconstruction connection. But $\tilde{u}, \tilde{v} \in(\operatorname{Ker} T \pi)^{\perp}$, so that

$$
\begin{aligned}
\tilde{u}, \tilde{v} \in A^{\omega} \cap(\operatorname{Ker} T \pi)^{\perp} & =\left((\operatorname{Ker} T \pi)^{\perp} \cap \operatorname{KerTJ}\right)^{\omega} \cap(\operatorname{Ker} T \pi)^{\perp} \\
& =\left(\left((\operatorname{Ker} T \pi)^{\omega}\right)^{\perp}+\operatorname{Ker} T \pi\right) \cap(\operatorname{Ker} T \pi)^{\perp} .
\end{aligned}
$$

Using the modularity property and the fact that KerT $\pi$ is isotropic, that is, $\operatorname{Ker} T \pi \subset(\operatorname{Ker} T \pi)^{\omega}$, and, hence,

$$
(\operatorname{Ker} T \pi)^{\perp} \supset\left((\operatorname{Ker} T \pi)^{\omega}\right)^{\perp}
$$

we obtain that

$$
\tilde{u}, \tilde{v} \in A^{\omega} \cap(\operatorname{Ker} T \pi)^{\perp}=\left((\operatorname{Ker} T \pi)^{\omega}\right)^{\perp} \cap(\operatorname{Ker} T \pi)^{\perp}=\left((\operatorname{Ker} T \pi)^{\omega}\right)^{\perp},
$$

but this space is also isotropic, that is, it is contained in its symplectic orthogonal because of (4.44)

$$
\left((\operatorname{Ker} T \pi)^{\omega}\right)^{\perp}=\left((\operatorname{Ker} T \pi)^{\perp}\right)^{\omega} \subset\left(\left((\operatorname{Ker} T \pi)^{\omega}\right)^{\perp}\right)^{\omega} .
$$

Thus, $\left.\omega^{\prime}\right|_{\mathrm{D}}=0$.

(3) Finally, combining the two arguments (1) and (2), for any $u \in T_{y} E$ and $v \in \mathrm{D}(\mathrm{y}), \tilde{\mathrm{u}} \in A$ and $\tilde{v} \in\left((\operatorname{Ker} \mathrm{T} \pi)^{\omega}\right)^{\perp}$. But,

$$
A^{\omega}=\left((\operatorname{Ker} T \pi)^{\perp} \cap \operatorname{Ker} T J\right)^{\omega}=\left((\operatorname{Ker} T \pi)^{\omega}\right)^{\perp}+(\operatorname{Ker} T J)^{\omega},
$$

so that $\tilde{v} \in A^{\omega}$ and $\omega(\tilde{u}, \tilde{v})=0$.

Corollary 4.16. The infinitesimal geometric reconstruction phase is computed according to

$$
\xi_{\text {geom }}(y)=D_{\mu} \omega^{\prime}(y)
$$

where the transverse derivative $\mathrm{D}_{\mu} \omega^{\prime}$ is computed using Lemma 4.11. 
22 Abstract mechanical connection and Abelian reconstruction ...

\section{Application: resonant three-wave interaction}

The three-wave equations describe the resonant quadratic nonlinear interaction of three waves and are obtained as amplitude equations in an asymptotic reduction of primitive equations in optics, fluid dynamics, and plasma physics. It was first analyzed by Alber, Luther, Marsden, and Robbins in [2] and later in [7]. Here we only quote the results relevant for the definition of the connection and the computation of phases and refer the reader to [2] for the detailed description. Some results for the Poisson reduction obtained here (such expressions for the Casimirs $C_{1}$ and $C_{2}$ as well as formulas (5.11) for the reduced Poisson bracket and (5.15) for the reduced symplectic structure) are original and were not presented in [2]. We use the canonical Hamiltonian structure and ignore an alternative Lie-Poisson description of this system.

\subsection{The phase space and its Kähler structure}

The phase space $\mathrm{P}$ of the system is $\mathbb{C}^{3}$ with appropriately weighed standard Kähler structure. In particular, a $\gamma_{i}$-weighed canonical Poisson bracket on $\mathbb{C}^{3}$ is used. This bracket has the real and imaginary parts of each complex dynamic variable $q_{i}$ as conjugate variables. The corresponding symplectic structure is written as follows:

$$
\omega(z, w)=-\sum_{k} \frac{1}{s_{k} \gamma_{k}} \operatorname{Im}\left(z_{k} \bar{w}_{k}\right),
$$

where $z, w \in \mathrm{T}_{\mathrm{q}} \mathbb{C}^{3}$ and $s_{\mathrm{k}}$ are sign variables.

Similarly, define a weighted metric on $\mathrm{P}$

$$
\mathfrak{s}(z, w)=\sum_{k} \frac{1}{s_{k} \gamma_{k}} \operatorname{Re}\left(z_{k} \bar{w}_{k}\right),
$$

and the standard complex structure $\mathcal{J}(z)=i z$. The Kähler structure then contains $\mathfrak{s}$ and $\omega$ as real and imaginary parts, respectively.

\subsection{The symmetry group and momentum map}

Consider the action of an Abelian group $\mathrm{T}^{2}$ on $\mathbb{C}^{3}$ given by

$$
\left(q_{1}, q_{2}, q_{3}\right) \longmapsto\left(\exp ^{-i \xi^{1}} q_{1}, \exp ^{-i\left(\xi^{1}+\xi^{2}\right)} q_{2}, \exp ^{-i \xi^{2}} q_{3}\right)
$$

where $\xi=\left(\xi_{1}, \xi_{2}\right)$ is an element of the Lie algebra $t^{2} \equiv \mathbb{R}^{2}$. The vector fields of the infinitesimal transformations corresponding to $\xi_{1}, \xi_{2}$ are given by

$$
\begin{aligned}
\xi_{\mathrm{p}}^{1}(\mathrm{q}) & =\left(-i \xi_{1} \mathrm{q}_{1},-i \xi_{1} \mathrm{q}_{2}, 0\right), \xi_{\mathrm{p}}^{2}(\mathrm{q}) \\
& =\left(0,-i \xi_{2} \mathrm{q}_{2},-i \xi_{2} \mathrm{q}_{3}\right) \in \mathrm{T}_{\mathrm{q}} \mathbb{C}^{3} .
\end{aligned}
$$


Points of the form $\left(q_{1}, 0,0\right),\left(0, q_{2}, 0\right),\left(0,0, q_{3}\right)$ have nontrivial isotropy subgroups, and thus account for singularities in the reduced space, that is, as the action is not free, the reduced space fails to be a smooth manifold (cf. [9]). Henceforth, we ignore these points and restrict ourselves to the set of regular points in $\mathbb{C}^{3}$.

The momentum map for this action was computed in [2] and is given by

$$
J\left(q_{1}, q_{2}, q_{3}\right)=\left(K_{1}, K_{2}\right)=\left(\frac{1}{2}\left(\frac{\left|q_{1}\right|^{2}}{s_{1} \gamma_{1}}+\frac{\left|q_{2}\right|^{2}}{s_{2} \gamma_{2}}\right), \frac{1}{2}\left(\frac{\left|q_{2}\right|^{2}}{s_{2} \gamma_{2}}+\frac{\left|q_{3}\right|^{2}}{s_{3} \gamma_{3}}\right)\right) \text {. }
$$

We keep the notations $\left(\mathrm{K}_{1}, \mathrm{~K}_{2}\right)$ for the values of the momentum map to be consistent with [9]; they play the role of $\mu=J(x)$ which was used throughout the paper.

It is checked directly using (5.3) that the momentum map is G-invariant. For further applications, we note that even though $J$ is not analytic, we can consider its differential as a real-valued map of the tangent space $T \mathbb{C}^{3}$ to $T \mathbb{R}^{2}$.

\subsection{The Hamiltonian}

The Hamiltonian for the three-wave interaction is

$$
\mathrm{H}=-\frac{1}{2}\left(\overline{\mathrm{q}}_{1} \mathrm{q}_{2} \overline{\mathrm{q}}_{3}+\mathrm{q}_{1} \overline{\mathrm{q}}_{2} \mathrm{q}_{3}\right) \text {. }
$$

Hamilton's equations are $\dot{\mathrm{q}}_{\mathrm{k}}=\left\{\mathrm{q}_{\mathrm{k}}, \mathrm{H}\right\}$ and it is straightforward to check that in complex notations they are given by

$$
\frac{d q_{k}}{d t}=-2 i s_{k} \gamma_{k} \frac{\partial H}{\partial \bar{q}_{k}} \text {. }
$$

\subsection{Poisson reduction}

It was shown in [2] that the following quantities constitute invariants for the $\mathrm{T}^{2}$ action

$$
X+i Y=q_{1} \bar{q}_{2} q_{3}, \quad Z_{1}=\left|q_{1}\right|^{2}-\left|q_{2}\right|^{2}, \quad Z_{2}=\left|q_{2}\right|^{2}-\left|q_{3}\right|^{2} .
$$

They provide coordinates for the four-dimensional (real) orbit space. The symplectic leaves in it are two-dimensional. This follows from the leaf correspondence theorem, as $\mathrm{T}^{2}$ is Abelian, and each point in it being a coadjoint orbit has co-dimension 2. One can define two Casimirs on $\mathbb{C}^{3} / \mathrm{T}^{2}$, for example,

$$
C_{1}=\left(X^{2}+Y^{2}\right)-K_{4}\left(2 s_{2} \gamma_{2} K_{1}+Z_{1}\right)\left(2 s_{3} \gamma_{3} K_{2}+Z_{2}\right)\left(2 s_{2} \gamma_{2} K_{2}-Z_{2}\right) \text {, }
$$

where $k_{4}=\left(s_{1} \gamma_{1} s_{2} \gamma_{2} s_{3} \gamma_{3}\right) /\left(s_{1} \gamma_{1}+s_{2} \gamma_{2}\right)\left(s_{2} \gamma_{2}+s_{3} \gamma_{3}\right)^{2}$, and

$$
C_{2}=\left(Z_{1}-2 s_{1} \gamma_{1} K_{1}\right)\left(s_{2} \gamma_{2}+s_{3} \gamma_{3}\right)+\left(Z_{2}+2 s_{3} \gamma_{3} K_{2}\right)\left(s_{1} \gamma_{1}+s_{2} \gamma_{2}\right) \text {, }
$$


24 Abstract mechanical connection and Abelian reconstruction ...

which can be obtained by a pull-back of properly defined Casimirs on $\mathfrak{g}^{*}$ using ideas of dual pairs (cf. [4, 13]). The level set of these Casimirs, defined for any momentum map value $\left(\mathrm{K}_{1}, \mathrm{~K}_{2}\right) \in \mathrm{U}$, where $\mathrm{U} \equiv \mathrm{J}\left(\mathbb{C}^{3}\right) \subset \mathrm{t}^{2}$, by the set $\left\{C_{1}=0, C_{2}=0\right\}$ determines the corresponding symplectic leaf $P_{\left(K_{1}, K_{2}\right)}$ in the reduced space.

The reduced Poisson bracket on $\mathbb{C}^{3} / T^{2} \cong\left(X, Y, Z_{1}, Z_{2}\right)$ is given for any two functions $f, k$ by

$$
\{\mathrm{f}, \mathrm{k}\}=\operatorname{det}\left(\nabla \mathrm{C}_{2} \nabla \mathrm{C}_{1} \nabla \mathrm{f} \nabla \mathrm{k}\right) .
$$

The reduced Hamiltonian equations of motion have the following form:

$$
\begin{aligned}
& \dot{X}=0, \quad \dot{Y}=-\frac{\partial C_{2}}{\partial Z_{2}} \frac{\partial C_{1}}{\partial Z_{1}}+\frac{\partial C_{2}}{\partial Z_{1}} \frac{\partial C_{1}}{\partial Z_{2}}, \\
& \dot{Z}_{1}=\frac{\partial C_{2}}{\partial Z_{2}} \frac{\partial C_{1}}{\partial Y}, \quad \dot{Z}_{1}=-\frac{\partial C_{2}}{\partial Z_{1}} \frac{\partial C_{1}}{\partial Y} .
\end{aligned}
$$

Notice that the second Casimir $C_{2}$ establishes a linear dependence between $Z_{1}$ and $Z_{2}$; hence, we can solve for one of them, say $Z_{1}$ and restrict ourselves to the consideration of three-dimensional subspace in $\mathbb{C}^{3} / T^{2}$ defined by $\left(X, Y, Z_{2}\right)$. The dynamics in $Z_{1}$ can then be trivially reconstructed. In this case, the first Casimir $C_{1}$ can be rewritten as

$$
\phi=\left(s_{2} \gamma_{2}+s_{3} \gamma_{3}\right)\left[\left(X^{2}+Y^{2}\right)-k_{3}\left(\delta-Z_{2}\right)\left(2 s_{3} \gamma_{3} K_{2}+Z_{2}\right)\left(2 s_{2} \gamma_{2} K_{2}-Z_{2}\right)\right]
$$

where $\kappa_{3}=\left(s_{1} \gamma_{1} s_{2} \gamma_{2} s_{3} \gamma_{3}\right) /\left(s_{2} \gamma_{2}+s_{3} \gamma_{3}\right)^{3}$ and $\delta=2 s_{2} \gamma_{2} K_{1}+2 s_{3} \gamma_{3}\left(K_{1}-\right.$ $\mathrm{K}_{2}$ ). This relation defines two-dimensional (perhaps singular) surfaces in $\left(X, Y, Z_{2}\right)$ space, with $Z_{1}$ determined by the values of the invariants and conserved quantities. These surfaces are called three-wave surfaces.

The reduced Poisson bracket in $\left(X, Y, Z_{2}\right)$ space is given by

$$
\{\mathrm{f}, \mathrm{k}\}=\nabla \phi \cdot(\nabla \mathrm{f} \times \nabla \mathrm{k})
$$

for any functions $f, k$. For a nonsingular point $y$ on a symplectic leaf $P_{\left(K_{1}, K_{2}\right)}$ the induced symplectic form is then given by

$$
\omega_{\left(K_{1}, K_{2}\right)}(v, w)=-\frac{\nabla \phi}{\|\nabla \phi\|^{2}} \cdot(v \times w),
$$

where $v, w \in \mathrm{T}_{y} \mathrm{P}_{\left(\mathrm{K}_{1}, \mathrm{~K}_{2}\right)}$; here $\left(\mathrm{K}_{1}, \mathrm{~K}_{2}\right)$ is the momentum value which determines a particular symplectic leaf $P_{\left(K_{1}, K_{2}\right)}$. Thus, for a function $f$ on the orbit space $\left(X, Y, Z_{2}\right)$, the corresponding Hamiltonian vector field has the form $X_{f}=-\nabla \phi \times \nabla f$.

The reduced Hamiltonian for the three-wave interaction is given by $h=$ $-X$ and produces the following reduced equations of motion:

$$
\dot{X}=0, \quad \dot{Y}=\frac{\partial \phi}{\partial Z_{2}}, \quad \dot{Z}_{2}=-2\left(s_{2} \gamma_{2}+s_{3} \gamma_{3}\right) Y
$$


which otherwise can be obtained by the restriction of the equations of motion in $\mathbb{C}^{3} / \mathrm{T}^{2} \cong\left(\mathrm{X}, \mathrm{Y}, \mathrm{Z}_{1}, \mathrm{Z}_{2}\right)$ to three-wave surfaces.

\subsection{Abstract mechanical connection}

First of all, we compute the locked inertia tensor $\mathbb{I}(q): \mathbb{R}^{2} \mapsto \mathbb{R}^{2}$ using its definition (4.2). It is an isomorphism for regular points $\mathrm{q}$ and is given by the following expression:

$$
\mathbb{I}(q)=\left(\begin{array}{cc}
2 K_{1} & \frac{\left|q_{2}\right|^{2}}{s_{2} \gamma_{2}} \\
\frac{\left|q_{2}\right|^{2}}{s_{2} \gamma_{2}} & 2 K_{2}
\end{array}\right),
$$

where $\mathrm{K}_{1}\left(\mathrm{q}_{1}, \mathrm{q}_{2}, \mathrm{q}_{3}\right)$ and $\mathrm{K}_{2}\left(\mathrm{q}_{1}, \mathrm{q}_{2}, \mathrm{q}_{3}\right)$ are components of the momentum map given by (5.5). Notice that II can be dropped to the quotient space:

$$
\mathbb{I}\left(X, Y, Z_{1}, Z_{2}\right)=\left(\begin{array}{cc}
2 K_{1} & \frac{2 s_{3} \gamma_{3} K_{2}+Z_{2}}{s_{2} \gamma_{2}+s_{3} \gamma_{3}} \\
\frac{2 s_{3} \gamma_{3} K_{2}+Z_{2}}{s_{2} \gamma_{2}+s_{3} \gamma_{3}} & 2 K_{2}
\end{array}\right),
$$

where $K_{1}, K_{2}$ are now functions of $\left(X, Y, Z_{1}, Z_{2}\right)$ as the momentum map factors through the quotient and are constant on each symplectic leaf, or a three-wave surface of thereof, in the reduced space.

Using Corollary 4.5, we can explicitly construct the corresponding abstract mechanical connection one-form

$$
\mathcal{A}(x) \cdot w=-\mathbb{I}^{-1}(x) \cdot \operatorname{Im}\left(\begin{array}{l}
\frac{\overline{\mathrm{q}}_{1} w_{1}}{\mathrm{~s}_{1} \gamma_{1}}+\frac{\overline{\mathrm{q}}_{2} w_{2}}{\mathrm{~s}_{2} \gamma_{2}} \\
\frac{\overline{\mathrm{q}}_{2} w_{2}}{\mathrm{~s}_{2} \gamma_{2}}+\frac{\overline{\mathrm{q}}_{3} w_{3}}{\mathrm{~s}_{3} \gamma_{3}}
\end{array}\right) .
$$

Comparing expression (5.19) with the definition of the Riemannian structure (5.2), we can conclude that

$$
\begin{aligned}
w \in \operatorname{hor}(\mathrm{q}) & \Longleftrightarrow\left\{\begin{array}{l}
\operatorname{Im}\left(\frac{\overline{\mathrm{q}}_{1} w_{1}}{\mathrm{~s}_{1} \gamma_{1}}+\frac{\overline{\mathrm{q}}_{2} w_{2}}{\mathrm{~s}_{2} \gamma_{2}}\right)=0 \\
\operatorname{Im}\left(\frac{\overline{\mathrm{q}}_{2} w_{2}}{\mathrm{~s}_{2} \gamma_{2}}+\frac{\overline{\mathrm{q}}_{3} w_{3}}{\mathrm{~s}_{3} \gamma_{3}}\right)=0
\end{array}\right. \\
& \Longleftrightarrow\left\{\begin{array}{l}
\mathfrak{s}\left(\xi_{\mathrm{p}}^{1}(\mathrm{q}), w\right)=0 \\
\mathfrak{s}\left(\xi_{\mathrm{p}}^{2}(\mathrm{q}), w\right)=0
\end{array}\right.
\end{aligned}
$$

that is, the horizontal space is precisely the metric orthogonal to the group orbits. Similar computations show that $(\operatorname{hor}(q))^{\omega}$ is determined by the span 
of vectors $\mathcal{J}\left(\xi_{p}^{1}(q)\right), \mathcal{J}\left(\xi_{p}^{2}(q)\right)$, and

$$
(\operatorname{hor}(\mathbf{q}))^{\omega}=\operatorname{span}\left\{i \xi_{p}^{1}(q), i \xi_{p}^{2}(q)\right\}=(\operatorname{Ker} T J(q))^{\perp} .
$$

The distribution $D=\widehat{A}$ on the symplectic stratification $j: P / G \rightarrow \mathfrak{g}^{*}$ is obtained by applying the tangent map $T \pi$ to the space $(\operatorname{hor}(q))^{\omega}$. Define vectors $v_{1}, v_{2}$ tangent to the quotient space at the point $\left(X, Y, Z_{1}, Z_{2}\right)$ to be the images of a basis in (hor $(\mathbf{q}))^{\omega}$ under this tangent map:

$$
\begin{aligned}
& v_{1}=T \pi(q)\left(i \xi_{P}^{1}\right)=2 \xi^{1} \cdot\left(X, Y, Z_{1}, \frac{s_{2} \gamma_{2}}{s_{2} \gamma_{2}+s_{3} \gamma_{3}}\left(2 s_{3} \gamma_{3} K_{2}+Z_{2}\right)\right) \\
& v_{2}=T \pi(q)\left(i \xi_{P}^{2}\right)=2 \xi^{2} \cdot\left(X, Y,-\frac{s_{2} \gamma_{2}}{s_{1} \gamma_{1}+s_{2} \gamma_{2}}\left(2 s_{1} \gamma_{1} K_{1}-Z_{1}\right), Z_{2}\right) .
\end{aligned}
$$

Then,

$$
\mathrm{D}\left(\mathrm{X}, \mathrm{Y}, \mathrm{Z}_{1}, \mathrm{Z}_{2}\right)=\mathrm{T} \pi(\operatorname{hor}(\mathrm{q}))^{\omega}=\operatorname{span}\left\{\boldsymbol{v}_{1}, \boldsymbol{v}_{2}\right\} .
$$

To finish the construction of the map L given by Lemma 4.11, we need to substitute $\mathbb{I}^{-1} \cdot v, \forall v \in\left(t^{2}\right)^{*}$ for the $\xi$. Then, for any $v=\sum v_{k} e^{k} \in\left(t^{2}\right)^{*}$, where $e^{k}$ is the dual basis of $\left(t^{2}\right)^{*}$, the map L is given by

$$
\mathrm{L}: \boldsymbol{v} \longmapsto \mathrm{L}(\boldsymbol{v})=\sum v_{\mathrm{k}} \mathrm{T} \pi\left(i\left(\mathbb{I}^{-1} \cdot v\right)_{\mathrm{P}}^{\mathrm{k}}\right)=\sum \boldsymbol{v}_{\mathrm{k}} \boldsymbol{v}_{\mathrm{k}}
$$

where in the expressions for $v_{k}$ we take $\xi^{k}=\left(\mathbb{I}^{-1} \cdot v\right)^{k}$.

\subsection{Phases for the three-wave interaction}

Recall that the reduced Hamiltonian on $P / G$ is $h=-X$. Applying Theorem 4.12, we can immediately obtain the $v$-component the associated dynamic phase by computing directional derivatives of the reduced Hamiltonian in the directions $\boldsymbol{v}=\boldsymbol{v}_{1}+\boldsymbol{v}_{2}$ in the transverse distribution $\mathrm{D}$ :

$$
\left\langle v, \xi_{\text {dyn }}\right\rangle=\operatorname{dh}(v)=\frac{2 h}{\operatorname{det} \mathbb{I}}\left(2 K_{1} v_{2}+2 K_{2} v_{1}-\frac{2 s_{3} \gamma_{3} K_{2}+Z_{2}}{s_{2} \gamma_{2}+s_{3} \gamma_{3}}\left(v_{1}+v_{2}\right)\right)
$$

where $\left(K_{1}, K_{2}\right)$ are the momentum values at $\left(X, Y, Z_{1}, Z_{2}\right)$ along the reduced trajectory $y_{t}$. To get the dynamic phase $g_{d y n}$, one integrates the exponent of this expression along the reduced trajectory $y_{t}$ on a three-wave surface.

The infinitesimal geometric phase $\xi_{\text {geom }}$, as a two-form on the reduced space, can be computed using (4.48), so that its $v$-component is given by

$$
\left\langle v, \xi_{\text {geom }}(y)\right\rangle=\left\langle v, D_{\mu} \omega^{\prime}(y)\right\rangle \text {. }
$$

This expression should be computed using standard formulas for the differentials of $p$-forms. We omit here the calculations of the dynamic phase as they crucially depend on the area over which the two-form is integrated. 


\section{Concluding remarks}

If the phase space $P$ has an almost Kähler structure, a preferred connection can be defined by declaring horizontal spaces at each point to be metric orthogonal to the tangent to the group orbit. We call it abstract mechanical connection. Then, explicit formulas for the corresponding $\mathfrak{g}^{*}$-valued oneform $\mathcal{A}$ in terms of the momentum map, symplectic and complex structures can be derived. Also, we show that in this case the horizontal spaces for the induced connections are metric orthogonal to the corresponding natural vertical spaces for each foliation.

These results are applied to the resonant three-wave interaction problem (cf. [2]). The corresponding horizontal spaces are constructed and a formula for the dynamic phase is obtained. The associated geometric phase is given by the integral of a two-form which is defined by the reduced symplectic structure.

\section{Acknowledgements}

We would like to extend our gratitude to Anthony D. Blaom for his time and invaluable help in the preparation of this manuscript; his input was crucial in developing some key ideas discussed here. We also would like to thank Tudor Ratiu and Sameer Jalnapurkar for helpful comments.

\section{References}

[1] M. Adams and T. Ratiu, The three-point vortex problem: commutative and noncommutative integrability, Hamiltonian Dynamical Systems (Boulder, C0, 1987), Amer. Math. Soc., Rhode Island, 1988, pp. 245-257. MR 90e:58041.

[2] M. S. Alber, G. G. Luther, J. E. Marsden, and J. M. Robbins, Geometric phases, reduction and Lie-Poisson structure for the resonant three-wave interaction, Phys. D 123 (1998), no. 1-4, 271-290. MR 2000g:37110.

[3] A. D. Blaom, Reconstruction phases via Poisson reduction, Differential Geom. Appl. 12 (2000), no. 3, 231-252. CMP 1764331.

[4] A A geometric setting for Hamlitonian perturbation theory, to appear, 2001.

[5] P. Heinzner and F. Loose, Reduction of complex Hamiltonian G-spaces, Geom. Funct. Anal. 4 (1994), no. 3, 288-297. MR 95j:58050. Zbl 816.53018.

[6] S. Kobayashi and K. Nomizu, Foundations of Differential Geometry. Vol I, Interscience Publishers, a division of John Wiley \& Sons, New York, 1963. MR 27\#2945.

[7] G. G. Luther, M. S. Alber, J. E. Marsden, and J. M. Robbins, Geometric nonlinear theory of quasi-phase-matching, J. Optical Soc. Am. B 17 (2000), 932-941.

[8] J. Marsden, R. Montgomery, and T. Ratiu, Reduction, symmetry, and phases in mechanics, Mem. Amer. Math. Soc. 88 (1990), no. 436, 110. MR 91b:58074. Zbl 713.58052. 
28 Abstract mechanical connection and Abelian reconstruction ...

[9] J. E. Marsden and T. S. Ratiu, Introduction to Mechanics and Symmetry: a Basic Exposition of Classical Mechanical Systems, Texts in Applied Mathematics, vol. 17, Springer-Verlag, New York, 1994. MR 95i:58073. Zbl 811.70002.

[10] _ Mechanics and Symmetry: Reduction Theory, Texts in Applied Mathematics, Springer, 1999.

[11] J. E. Marsden, T. S. Ratiu, and J. Scheurle, Reduction theory and the LagrangeRouth equations, J. Math. Phys. 41 (2000), no. 6, 3379-3429. CMP 1768627.

[12] M. Otto, A reduction scheme for phase spaces with almost Kähler symmetry. Regularity results for momentum level sets, J. Geom. Phys. 4 (1987), no. 2, 101-118. MR 89k:58104. Zbl 642.53040.

[13] A. Weinstein, The local structure of Poisson manifolds, J. Differential Geom. 18 (1983), no. 3, 523-557. MR 86i:58059. Zbl 524.58011.

Sergey Pekarsky: Control and Dynamical Systems, 107-81, California Institute of Technology, Pasadena, CA 91125, USA

E-mail address: sergy@cds.caltech.edu

Jerrold E. Marsden: Control and Dynamical Systems, 107-81, California Institute of Technology, Pasadena, CA 91125, USA

E-mail address: marsden@cds.caltech.edu 


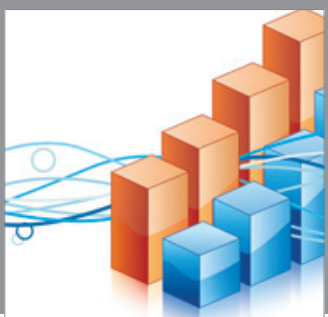

Advances in

Operations Research

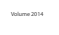

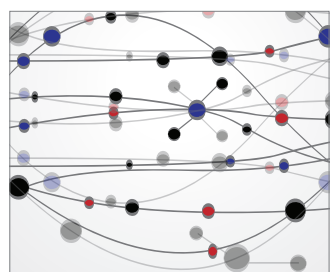

\section{The Scientific} World Journal
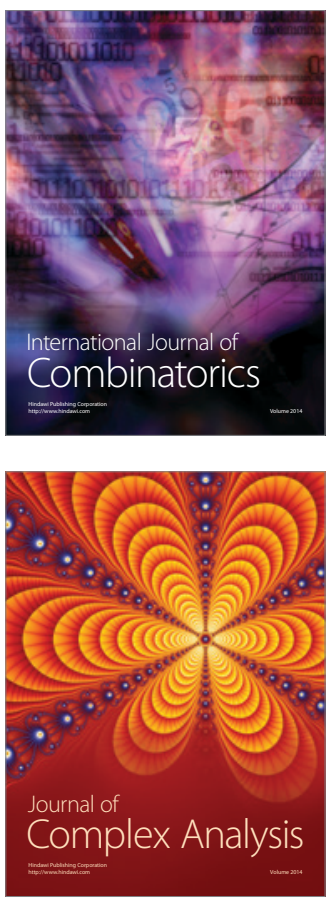

International Journal of

Mathematics and

Mathematical

Sciences
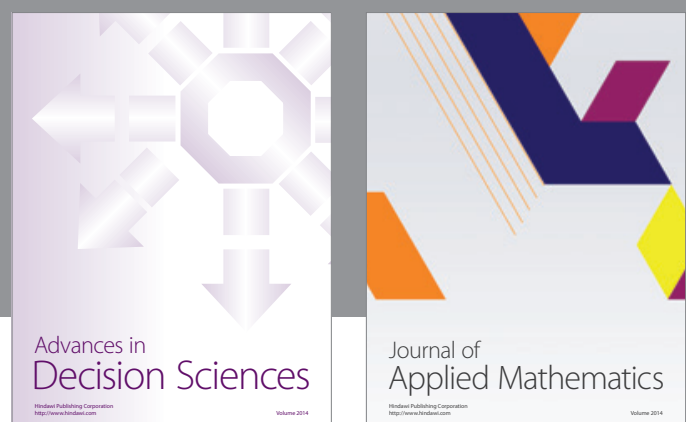

Journal of

Applied Mathematics
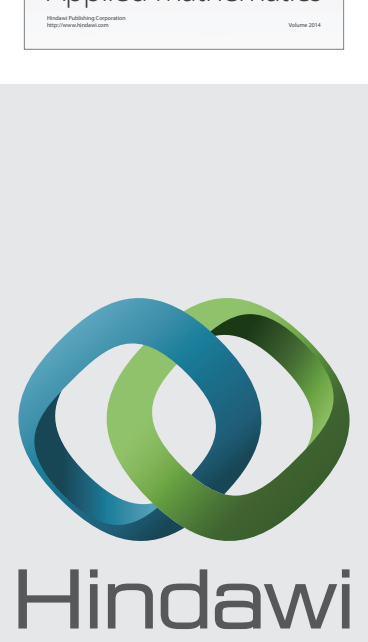

Submit your manuscripts at http://www.hindawi.com
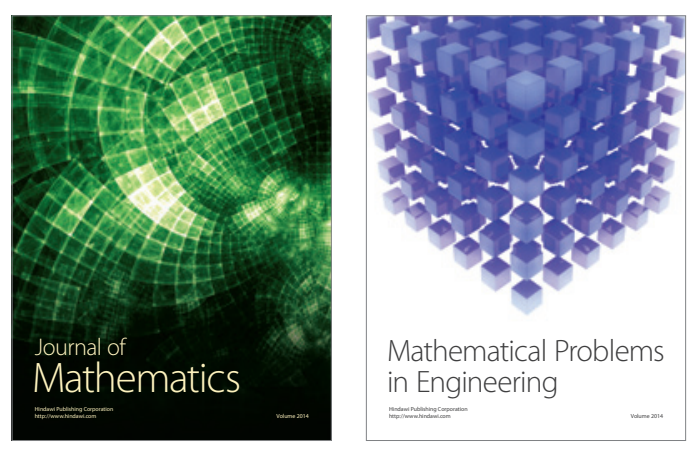

Mathematical Problems in Engineering
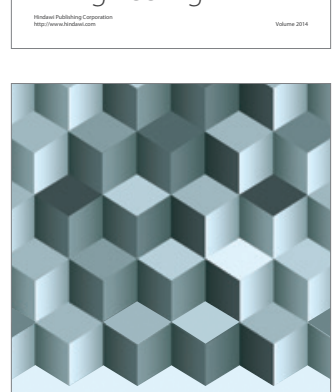

Journal of

Function Spaces
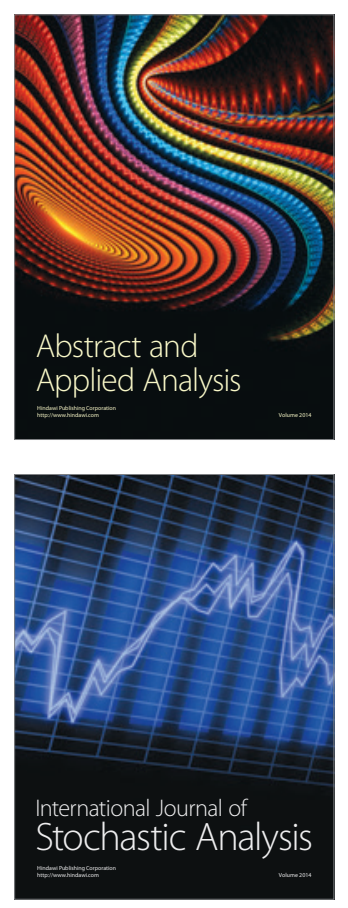

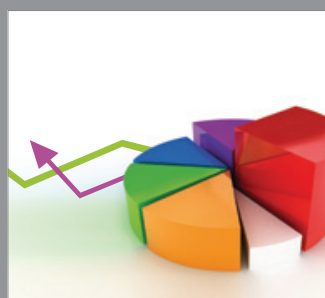

ournal of

Probability and Statistics

Promensencen
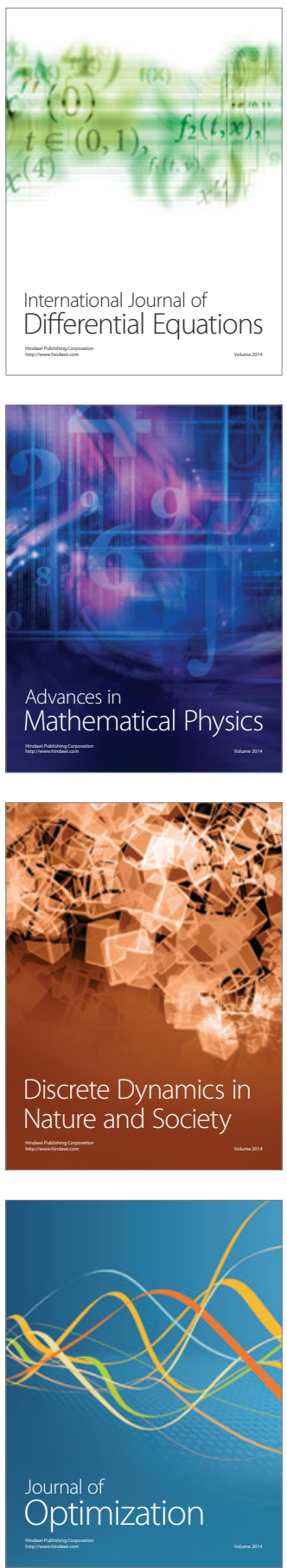